\title{
Child distress during immunization: The influence of child and parent individual difference variables
}

\author{
Melissa DeMore \\ West Virginia University
}

Follow this and additional works at: https://researchrepository.wvu.edu/etd

\section{Recommended Citation}

DeMore, Melissa, "Child distress during immunization: The influence of child and parent individual difference variables" (2006). Graduate Theses, Dissertations, and Problem Reports. 2504.

https://researchrepository.wvu.edu/etd/2504

This Dissertation is protected by copyright and/or related rights. It has been brought to you by the The Research Repository @ WVU with permission from the rights-holder(s). You are free to use this Dissertation in any way that is permitted by the copyright and related rights legislation that applies to your use. For other uses you must obtain permission from the rights-holder(s) directly, unless additional rights are indicated by a Creative Commons license in the record and/ or on the work itself. This Dissertation has been accepted for inclusion in WVU Graduate Theses, Dissertations, and Problem Reports collection by an authorized administrator of The Research Repository @ WVU.

For more information, please contact researchrepository@mail.wvu.edu. 
Child Distress during Immunization:

The Influence of Child and Parent Individual Difference Variables

\author{
Melissa DeMore, M.A. \\ Dissertation submitted to the \\ College of Arts and Sciences \\ at West Virginia University \\ in partial fulfillment of the requirements \\ for the degree of \\ Doctor of Philosophy \\ in \\ Clinical Child Psychology
}

Kevin Larkin, Ph.D, Chair

Cheryl McNeil, Ph.D.

Katherine Karraker, Ph.D.

Steven Branstetter, Ph.D.

Department of Psychology

Shiv Someshwar, M.D.

Department of Pediatrics

Morgantown, West Virginia University

2006

Keywords: Pediatric, Distress, Immunizations

Copyright 2006 Melissa DeMore 


\begin{abstract}
Child Distress during Immunization:

The Influence of Child and Parent Individual Difference Variables
\end{abstract}

\title{
Melissa DeMore
}

Routine childhood immunization injections result child distress, which may have lasting negative effects on children, parents, and staff, and impact adherence to schedules of immunization. Aims of the current study were to (a) examine the role of child and parent individual difference variables in relation to child immunization distress, and (b) determine whether child distress predicts future immunization schedule adherence.

Parents of 50 children (aged 12 - 18 months) attending a visit to a pediatric medical clinic for purposes of immunization completed measures of child temperament, parent psychopathology, immunization beliefs, and prior medical distress of the child. Child distress during the immunization injection was measured via parent and nurse ratings and a behavior observational scale. Adherence to the immunization schedule was assessed via the Immunization Delivery Effectiveness Score (IDEA) and the families' attendance at a followup immunization appointment.

Positive correlations were observed between child distress and prior medical distress of the child, difficult child temperament, and parent psychopathology. Negative correlations were found between child distress and child age and parent immunization beliefs. Boys exhibited more distress than girls. Child age and parent psychopathology each made unique contributions in explaining the variance in child distress. An interaction was observed between a) prior medical experience and immunization adherence, and b) health care attitudes and immunization adherence in the relation to child distress. Magnitude of child distress was greatest among children (a) with prior negative medical experiences and poorer adherence to schedules of immunization, and (b) with parents possessing negative immunization beliefs and poorer adherence to schedules of immunization. Child distress did not predict attendance at a subsequent clinic visit for purposes of immunization. 
Several child and parent factors are related to various measures of child distress employed in this study. Results offer partial support for extant literatures (i.e., relations among immunization distress and age, gender, negative prior medical experiences, healthcare attitudes), and expand on a dearth in literature (i.e., relation between child distress and parent psychopathology). Findings may assist clinicians in identifying children at greatest risk for experiencing significant distress reactions during immunizations and providing training in effective interventions designed to minimize pain. 


\section{$\underline{\text { Acknowledgments }}$}

I would like to thank Kevin Larkin, Ph.D., for the generous amount of time and effort he put forth as my dissertation chair as well as my academic advisor. Kevin agreed to serve as both my dissertation chair and academic advisor in the absence of my previous advisor. This is but one demonstration of the level of support Kevin has provided in academic training. I also would like to thank Katherine Karraker, Ph.D., Cheryl McNeil, Ph.D., Shiv Someswhar, M.D., and Steven Branstetter, Ph.D. for the attention and time they devoted toward my dissertation, as members of my dissertation committee. Also deserving of appreciative recognition is Andy Lopez-Williams, Ph.D. whose comments on my dissertation, as a former dissertation committee member, were helpful. The contributions to my dissertation made by all of my committee members have been invaluable.

I acknowledge and thank Shiv Someswhar, M.D., and the nursing staff at the Pediatric and Adolescent Group Practice clinic at the Health Sciences Center of West Virginia University for supporting and encouraging my research interests and allowing me to recruit their patients. Many thanks to the undergraduate research assistants who aided me in participant recruitment and data entry. A special gratitude goes to my family and friends for their immeasurable encouragement, support, and patience during my doctoral dissertation work.

Finally, I greatly appreciate financial assistance for my dissertation provided from the Doctoral Research Award (Eberly College of Arts and Sciences at West Virginia University). The financial contribution of this award covered the bulk of expenses related to participant payment. 
Table of Contents

Title Page $\quad$ i

$\begin{array}{ll}\text { Abstract } & \text { ii }\end{array}$

Acknowledgements $\quad$ iv

Table of Contents $\quad$ v

$\begin{array}{ll}\text { List of Tables } & \text { ix }\end{array}$

List of Figures $\quad$ xii

Introduction $\quad 1$

Description of Pain 3

$\begin{array}{ll}\text { Pediatric Immunizations } & 4\end{array}$

Importance of Pediatric Procedural Pain Management 5

Individual Difference Variables Related to Distress during Procedural Pain $\quad 7$

History of Procedural Distress $\quad 8$

$\begin{array}{ll}\text { Age and Gender } & 11\end{array}$

$\begin{array}{ll}\text { Child Temperament } & 15\end{array}$

Parent Health Care Attitudes 17

$\begin{array}{ll}\text { Parent Psychopathology } & 19\end{array}$

Adherence to Immunization Schedules $\quad 20$

Statement of Purpose and Significance of the Study 22

$\begin{array}{ll}\text { Method } & 26\end{array}$

$\begin{array}{ll}\text { Participants } & 26\end{array}$

$\begin{array}{ll}\text { Measures } & 27\end{array}$

Patient Information Form $\quad 27$ 
$\begin{array}{ll}\text { Visual Analogue Scales } & 28\end{array}$

$\begin{array}{lr}\text { Toddler Temperament Scale } & 29\end{array}$

Health Care and Injection Attitudes Questionnaire 30

Brief Symptom Inventory 31

$\begin{array}{ll}\text { Immunization Schedule Adherence } & 31\end{array}$

Reasons for Nonattendance Form $\quad 33$

$\begin{array}{ll}\text { Instrumentation } & 33\end{array}$

$\begin{array}{ll}\text { Experimental Environment } & 33\end{array}$

$\begin{array}{ll}\text { Procedure } & 34\end{array}$

$\begin{array}{ll}\text { Results } & 35\end{array}$

$\begin{array}{ll}\text { Preliminary Analyses } & 35\end{array}$

Descriptive Statistics for Demographic Variables $\quad 37$

Aim One: Individual Difference Variables and Measures of Child Distress during 12

- 18 month immunizations $\quad 38$

$\begin{array}{ll}\text { Past Medical Distress } & 40\end{array}$

$\begin{array}{ll}\text { Child Age and Gender } & 40\end{array}$

$\begin{array}{ll}\text { Child Temperament } & 40\end{array}$

$\begin{array}{ll}\text { Parent Health Care Attitudes } & 41\end{array}$

$\begin{array}{ll}\text { Parent Psychopathology } & 41\end{array}$

Adherence to Immunization Schedules $\quad 42$

Relations between Child Distress and Individual Difference Variables $\quad 42$

Aim Two: Interaction Variables among Individual Difference Variables and 
Measures of Child Distress during 12 - 18 Month Immunizations

Past Medical Distress and Adherence to Immunization Schedules

Parent Health Care Attitudes and Adherence to Immunization Schedules

Parent Psychopathology and Adherence to Immunization Schedules

Aim Three: Measures of Child Distress during 12 - 18 month Immunizations and

Attendance at Future Immunization Appointment

Discussion

Relation Between Past Medical Distress and Distress During 12 - 18 Month

Immunizations

Relation Between Child Age and Distress During 12 - 18 Month Immunizations

Relation Between Child Gender and Distress During 12 - 18 Month

Immunizations

Relation Between Child Temperament and Distress During 12 - 18 Month

Immunizations

Relation Between Parent Health Care Attitudes and Distress During 12 - 18 Month

Immunizations

Relation Between Parent Psychopathology and Distress During 12 - 18 Month

Immunizations

Relation Between Adherence to Immunization Schedules and Distress During 12 18 Month Immunizations

Measures of Child Distress During 12 - 18 Month Immunizations and Attendance at

Future Immunization Appointment 
Future Directions and Clinical Implications

References

Appendices

85

Tables

93

Figures

126 


\section{List of Tables}

Table 1. Frequencies (and Percentages) for Demographic Variables 93

Table 2. Means and Standard Deviations for Demographic Variables 97

Table 3. Descriptive Statistics for Measures of Distress 98

Table 4. Descriptive Statistics for Measures of Child Temperament 99

Table 5. Descriptive Statistics for Measures of Health Care Beliefs 100

Table 6. Descriptive Statistics for Measures of Parent Psychopathology 101

Table 7. Descriptive Statistics for Measures of Adherence to Immunization Schedule 102

Table 8. Correlations between Demographic Variables and Child Distress Variables 103

Table 9. Correlations between Child Temperament Scales and Child Distress

Variables

Table 10. Correlations between Measures of Health Care Beliefs and Child Distress

Variables

Table 11. Correlations between Measures of Parent Psychopathology and Child Distress

Variables

Table 12. Correlations between Measures of Adherence to Immunization Schedule and Child Distress Variables

Table 13. Multiple Regression Analysis with MBPS Score: Child Age, Child

Gender, Child Temperament, Parent Health Beliefs, Prior Medical Experience, and

Parent Psychopathology

Table 14. Multiple Regression Analysis with Parent Rating of Child Distress:

Child Age, Child Gender, Child Temperament, Parent Health Beliefs, Prior Medical

Experience, and Parent Psychopathology 
Table 15. Multiple Regression Analysis with Nurse Rating of Child Distress: Child Age, Child Gender, Child Temperament, Parent Health Beliefs, Prior Medical

Experience, and Parent Psychopathology

Table 16. Hierarchical Multiple Regression Analysis with MBPS Injection Score:

Interaction of Prior Medical Experience and Adherence to Immunization Schedule

Table 17. Hierarchical Multiple Regression Analysis with Parent Rating of Child

Distress: Interaction of Prior Medical Experience and Adherence to Immunization

Schedule

Table 18. Hierarchical Multiple Regression Analysis with Nurse Rating of Child

Distress: Interaction of Prior Medical Experience and Adherence to Immunization Schedule 114

Table 19. Hierarchical Multiple Regression Analysis with MBPS Injection Score:

Interaction of Parent Health Beliefs and Adherence to Immunization Schedule

Table 20. Hierarchical Multiple Regression Analysis with Parent Rating of Child

Distress: Interaction of Parent Health Beliefs and Adherence to Immunization

Schedule

Table 21. Hierarchical Multiple Regression Analysis with Nurse Rating of Child

Distress: Interaction of Parent Health Beliefs and Adherence to Immunization

Schedule

Table 22. Hierarchical Multiple Regression Analysis with MBPS Injection Score:

Interaction of Parent Psychopathology and Adherence to Immunization Schedule

Table 23. Hierarchical Multiple Regression Analysis with Parent Rating of Child

Distress: Interaction of Parent Psychopathology and Adherence to Immunization 
Schedule

Table 24. Hierarchical Multiple Regression Analysis with Nurse Rating of Child

Distress: Interaction of Parent Psychopathology and Adherence to Immunization

Schedule

Table 25. Correlations between Attendance at Follow-Up Visit and Demographic

Variables

Table 26. Hierarchical Logistic Regression Analysis Predicting Attendance at

Follow-Up Immunization Visit by MBPS Injection Score

Table 27. Hierarchical Logistic Regression Analysis Predicting Attendance at Follow-Up Immunization Visit by Parent Rating of Child Distress

Table 28. Hierarchical Logistic Regression Analysis Predicting Attendance at Follow-Up Immunization Visit by Nurse Rating of Child Distress 


\section{List of Figures}

Figure Captions

Figure 1. Interaction between Prior Medical Experience and Adherence to

Immunization Schedules: MBPS Injection Score

Figure 2. Interaction between Health Care Attitudes and Adherence to

Immunization Schedules: MBPS Injection Score

128 


\section{Child Distress during Immunization:}

\section{The Influence of Child and Parent Individual Difference Variables}

Pain is one of the most common human experiences, affecting nearly everyone irrespective of gender, race, age, or social class. However, the experience of pain varies greatly across individuals and situations, regarding its intensity, duration (e.g., chronic and acute pain), and the circumstances under which the pain occurs (e.g., pain related to medical procedures, injury, or illness). Research focusing on the experience of pain in infancy and childhood is important, as research suggests that early pain experiences may have a longterm impact on behaviors and beliefs regarding the experience of pain later in life (e.g., Pate, Blount, Cohen, \& Smith, 1996). Although both immediate and long-term negative outcomes have been associated with painful medical and dental procedures conducted on pediatric patients, research predicting the magnitude of distress associated with procedural pain among pediatric patients remains incomplete (for a review see Blount, Piira, \& Cohen, 2003). In brief, although a number of variables have been hypothesized to influence the magnitude of distress experienced during painful medical and dental procedures, we do not know which variables best explain the magnitude of these pain-elicited distress responses. Although research examining variables that are linked with these distress responses could prove valuable in predicting any experience of pediatric pain, it may be particularly beneficial to explore the prediction of distress in response to pediatric immunization pain, given that this is the most common type of painful medical procedure that almost all children encounter.

The identification of individual differences related to procedural distress is particularly important given the subjective nature of pain. That is, similar aversive stimuli 
are known to elicit vastly different pain responses among individuals. Several demographic and historical variables have been examined in predicting the magnitude of this pain response. For example, the experience of pain during past pediatric procedures has been found to predict increased pain and distress during subsequent pediatric medical procedures (Dalqhuist, Gil, Armstrong, DeLawyer, Greene, \& Wuori, 1986). Additionally, female gender (Hildegard \& LeBaron, 1982; Katz, Kellerman, \& Siegel, 1980) and a younger age (e.g., Schechter, Bernstein, Beck, Hart, \& Scherzer, 1991) have both been associated with increased distress during immunizations. Identification of other individual differences that predict distress in response to pediatric medical procedures, however, has been less successful. This dearth of research is unfortunate as this knowledge could allow health care professionals to identify children who are at risk for high levels of distress, and to develop prevention (e.g., education programs) or intervention efforts (e.g., pain management programs) to best meet the needs of those at-risk individuals. Additionally, the lack of research in this area limits the clinical application of proven pharmacological and behavioral interventions for pain management (for review see Piira, Hayes, \& Goodenough, 2002) that could be delivered efficaciously to at-risk individuals who need it the most.

This paper will begin by examining the construct of pain within the context of pediatric immunizations and will explore data as well as theoretical underpinnings regarding the link between several variables associated with distress during immunization procedures. This review of the literature serves as an introduction to the empirical study that follows that examines relations between variables hypothesized to be linked with the extent of distressing pain responses during immunization in a group of 12 - 18 month old children undergoing a routine immunization procedure in a medical clinic. 


\section{Description of Pain}

The International Association for the Study of Pain (IASP) offers the most commonly accepted definition of pain as "an unpleasant sensory and emotional experience associated with actual or potential tissue damage, or described in terms of such damage" (Merskey \& Bogduk, 1994, pp. 209 - 214). The IASP further characterizes pain as a subjective experience wherein the inability of an individual to communicate their experience of pain verbally does not negate the possibility that the individual is experiencing pain. As such, differential verbalizations of pain can be observed across individuals experiencing identical tissue damage or sensory stimulation. Presumably, this subjective nature of pain is affected by both biological and psychological factors. Biologically, individuals may exhibit different sensitivity to painful stimuli, including the sensitivity of the sensory receptors to painful stimuli, as well as differential responsivity of the afferent tracts that transmit pain messages from peripheral sites to the brain (Merskey \& Bogduk). Psychologically, individual variability in personal history of exposure to painful stimuli, differential exposure to models of responding to painful stimuli, and reinforcement/punishment associated with previous exposure to painful stimuli may each influence the verbal expression of pain (Merskey \& Bogduk).

Whereas pain is recognized as an experience that typically has a proximate physical cause, it can be reported in the absence of tissue damage or any likely pathophysiological cause (e.g., phantom limb pain). Therefore, the report of pain is not only associated with actual tissue damage, but can also be related to anticipated tissue damage or previous tissue damage (Merskey \& Bogduk, 1994). 
Although not explicitly stated, the IASP definition alludes to physiological, cognitive, and behavioral components of the experience of pain (Merskey \& Boduk, 1994). Specifically, physiological parameters such as heart rate, vagal tone, respiratory rate, blood pressure, palmar sweating, oxygen saturation, and intracranial pressure, have been shown to change in response to pain (Sweet \& McGrath, 1998). Cognitive components of pain can be more difficult to describe, although definitions such as Melzack's (1999), are more lucid and characterize pain as having a distinctly "unpleasant, affective quality." Generally, pain is conceptualized as being associated with emotions such as fear, distress, and annoyance. Behavioral manifestations of pain can last for several minutes after the introduction of a noxious stimulus and can include a wide range of behaviors. For example, individuals may react to noxious stimuli with grimacing, flailing, bulging of the brow, muscular rigidity, crying, or attempts to withdraw from the noxious stimuli (McGrath, 1990).

For many years, health care professionals expressed the belief that young children did not experience and/or remember pain in the same way as adults (Finley \& McGrath, 1998). Within the past several decades, however, there has been a shift in this attitude so that it is now known that young children and infants have the capacity to feel pain of varying intensity, and therefore are deserving of humane pain alleviation treatment. In addition, data is mounting to suggest that pain experienced in childhood has significant and possibly permanent negative psychological and physiological effects on individuals (for a review see Finley \& McGrath).

\section{Pediatric Immunizations}

The most widespread painful medical procedure of childhood, starting just a few days after birth, is a series of routine childhood immunizations (Reis, Roth, Syphan, Tarbell, \& 
Holubkov, 2003). In the United States (U.S.), the national childhood immunization schedule calls for healthy children to receive approximately 25 intra-muscular immunization injections by the time they are 6 years of age (Centers for Disease Control and Prevention [CDC], 2003). In addition, although many states allow for exemptions, most U.S. state legislatures require documentation indicating that children have received routine immunization injections prior to enrollment in public elementary schools (CDC).

There are many benefits to pediatric immunization. Vaccines help protect individuals from dangerous and deadly diseases. Numerous infectious diseases now have safe and effective vaccines (e.g., diphtheria). Although the spread of these often-fatal diseases has been well contained in much of the world through effective immunization programs, they have not been eliminated entirely and continue to pose a public health threat in many countries. Those who are not immunized can transmit vaccine-preventable disease throughout their communities; unvaccinated individuals are vulnerable to contracting these diseases and can spread them to other un-immunized individuals who are too young to have been fully immunized (i.e., infants), individuals who cannot be immunized for medical reasons (e.g., individuals with various immunologic diseases), and to children whose immunizations failed to provide immunity (CDC, 2003).

\section{Importance of Pediatric Procedural Pain Management}

Although many view childhood immunizations as relatively benign procedures, research indicates that a substantial proportion of children experience significant levels of distress during immunization procedures (Jacobson, Swan, Adegbenro, Ludington, Wollan, et al., 2001). Jacobsen and colleagues found that at least $20 \%$ of children aged 4 to 6 years and as many as $90 \%$ of children 15 to 18 months old exhibited levels of distress ranging from " 3 " to 
"5" on a 5-point Likert-type scale ( 5 = "worst possible distress") according to nurse's reports. In addition, results of a national telephone survey revealed that approximately $47 \%$ of U.S. children (under the age of 18 years) surveyed report that shots or needles are the things they dislike the most when attending a physician office visit (Princeton Survey Research Associates [PSRA], 1996). Likewise, 23\% of parents with children aged 13 years and younger have delayed or avoided some medical procedure for their child to avoid a pediatric immunization procedure during the same office visit (PSRA).

Although procedural pain appears to have a negative impact on adherence to immunization schedules, pediatric pain during a range of pediatric medical procedures is linked to numerous other immediate and long-term negative outcomes. In the short-term, poor pain management is associated with immediate, negative psychological outcomes for the child patient, parent, and clinic staff (for a review see Blount et al., 2003), including increased child reports of anxiety during the medical procedure (e.g., Jacobson et al., 2001), and collateral anxiety in the caregiver who accompanies the child to the immunization visit (Cohen, Blount, \& Panapoulos, 1997). The research regarding long-term effects of procedural pain documents impairment in physiological, behavioral, and cognitive areas of functioning. Studies with humans as well as rats indicate that physiological long-term consequences of inadequate pain management may include higher pain sensitivity during subsequent medical treatments (Anand, Coskun, Thrivikraman, Nemeroff, \& Plotsky, 1999; Taddio, Goldbach, Ipp, Stevens, \& Koren, 1995). Moreover, painful experiences in childhood have been linked to poorer health care attitudes, elevated fear of medical procedures, and avoidance of medical care (Pate et al., 1996), as well as greater anxiety and 
decreased cooperation with venipuncture procedures in adulthood (Bijttebier \& Vertommen, 1998).

In summary, pediatric distress related to immunization procedures may be related to negative medical and behavioral outcomes. Specifically, pediatric immunization distress may be associated with decreased adherence to immunization schedules (Meyerhoff, Weniger, \& Jacobs, 2001; Reis, 1997). More generally, childhood procedural distress is related to negative short-term outcomes for children, their caregivers, and medical staff (Blount et al., 2003) and to negative long-term outcomes for infants and children (e.g., Taddio et al., 1995). In comparison to the amount of knowledge regarding consequences of pain, there are relatively few studies exploring predictors of distress during childhood immunizations (Blount et al.). The literature does suggest, however, that there are several individual difference variables that are associated with children who may be more susceptible to laboratory-induced (e.g., cold pressor task) and medical procedure-related pain. The following section explores the potential role of each of these variables in relating to pediatric distress during immunizations.

Individual Difference Variables Relating to Distress during Procedural Pain

A few historical, demographic, and constitutional individual difference variables have been identified that bear some relation to predicting child distress during painful medical procedures like injections, including history of procedural distress (e.g., Dalqhuist et al., 1986; Lumley, Melamed, \& Abeles, 1993), child age and gender (e.g., Bachanas \& Roberts, 1995; Goodenough, Kampel, Champion, Laubreaux, Nicholas, Ziegler, \& McInerney, 1997; Schechter et al., 1991) and child temperament (e.g., Schechter et al.; Sweet, McGrath, \& Symons, 1999). Research pertaining to individual difference variables that have been 
associated with child distress during various medical procedures will be reviewed in the following section. In addition, the individual difference variables of parental health care attitudes, adherence to immunization schedules, and parent psychopathology, which can be hypothesized to be related to the magnitude of child distress during medical procedures, but have limited or no empirical support, will be introduced.

\section{History of Procedural Distress}

The contribution of previous pain experiences has long been thought to influence current pain (Cheng, Foster, \& Hester, 2003). Indeed, a maxim in psychology holds that, in general, one of the better predictors of future behavior is past behavior. Accordingly, the association between parent report of children's previous medical experiences and observed behavior during medical procedures has been examined. Congruent with expectations, findings indicate that reported distress during prior medical procedures is predictive of distress during future procedures (Dalqhuist et al., 1986).

Dalqhuist and colleagues (1986) examined 79 3- to 12-year-old children presenting at a pediatric outpatient clinic for a sore throat and subsequent throat culture exam (i.e., tonsils are swabbed with cotton). Children's parents were interviewed to ascertain the approximate number of previous throat cultures, medical appointments, dental appointments, and hospitalizations the child had experienced. Parents also rated their child's reactions to each of these procedures on a 7 -point Likert scale $(1=$ negative, $4=$ no reaction, $7=$ positive). Approximately half of the children were classified as having had a negative prior medical experience (i.e., a rating of " 3 " or below on any of the four procedures); the remaining children fell within a neutral/positive experience category (i.e., those who received ratings greater than or equal to " 4 " in all domains). This dichotomous 
classification resulted in a relatively even distribution of participants across groups.

Findings indicated that the quality of the child's previous medical experience was significantly, inversely associated with observed child distress, as well as with parental and physician ratings of child anxiety during the clinic procedure. Conversely, the number of previous medical examinations was not significantly related to the amount of observed child distress during a throat culture. This study confirms that the quality of children's prior medical experience, not the quantity of these visits, was related to child distress during later medical experiences.

The relation between prior pain experience and amount of observed child distress extends to different pediatric populations as well. Lumley, Melamed, and Abeles (1993) examined 50 children aged 4 to 10 years (and their mothers) who were undergoing elective ear, nose, or throat surgery. Maternal retrospective report of the quality of children's reactions to prior medical experiences (i.e., past operations, dental procedures, and general medical events) was assessed on three 7-point scales ("very negative" to "very positive"). Child distress behaviors were recorded in the operating room during pre-surgery procedures and prior to general anesthesia induction. Results suggested that a negative reaction to prior medical experiences was one of the best predictors of distress in children undergoing ear, nose, or throat surgery, again confirming an association between the quality of prior medical experience and child distress during surgery.

In addition to the association between prior medical experiences and child distress, prior experiences also appear to bear a significant relation to children's perception of pain (Harbeck \& Peterson, 1992). Harbeck and Peterson interviewed 500 children, adolescents, and young adults ranging in age from 3 to 23 years in order to assess understanding of pain 
from a developmental perspective. Participants were asked to endorse the number of pains they had experienced in the past from a list of three types of commonly reported pains (i.e., injection, headache, skinned knee), thus yielding a score of "frequency of mentioned pains." The number of child-endorsed pains correlated significantly and positively with their current perceptions of pain (e.g., their attributions of why they experienced pain). Whereas causality cannot be inferred from this correlational finding, it is congruent with previous work (Dalqhuist et al., 1986; Lumley et al., 1993).

In contrast to the aforementioned studies, others have found that the number of previous medical experiences was negatively correlated with distress in children undergoing bone marrow aspirations (Jay, Ozolins, Elliot, \& Caldwell, 1983), suggesting a habituation effect. In this population, Jay and colleagues examined the observed behavioral distress of 42 pediatric cancer patients undergoing bone marrow aspiration (BMA) procedures across three age groups ( 2 to 6 years, 7 to 12 years, and 13 to 20 years). In order to examine the degree to which children and adolescents habituated to these aversive procedures, two measures were employed: the number of previous BMAs received, and the number of months since diagnosis with cancer. Results indicated significant, negative correlations between each of the habituation measures and ratings of observed distress during BMA, suggesting that children and adolescents do exhibit less distress during BMA procedures as a function of the number of BMAs experienced. Moreover, these relations remained significant even after the effects of age were statistically controlled.

In a similar study, Katz and colleagues (1980) failed to find support for the aforementioned findings. These researchers evaluated the behavioral distress of 115 children and adolescents with cancer undergoing BMAs across three age groups (8 months 
to 6 years, 7 to 9 years, and 10 to 17 years). Time since diagnosis and time elapsed since last BMA were both examined in order to assess possible effects of habituation to BMAs across time. Results failed to find significant correlations between either of the habituation measures and observed distress. The reason for the discrepancy between Katz's findings and those of Jay and colleagues concerning habituation is unclear, but may be related to differences in clinic size, clinic procedures, staff-patient ratio, and other interpersonal variables. More research is obviously needed to examine the role of prior experience in pediatric behavioral distress during BMAs.

In summary, although higher parent ratings of children's previous pain reactions during medical procedures appear to be linked with increased distress during subsequent, minimally invasive procedures, the literature on previous exposure to more painful and prolonged medical procedures like BMAs is mixed. Due to the differences in BMA procedures and immunizations (e.g., greater intensity and duration of pain associated with BMAs as compared to intramuscular injections, immunization versus treating a lifethreatening disease), however, it is unlikely that identical findings would be expected across studies that examine these two quite distinct medical procedures.

\section{Age and gender}

A few demographic characteristics have been associated with procedural distress in pediatric patients, specifically age and gender. The majority of research concerning predictors of pediatric pain suggests that a child's chronological age is related to the level of behavioral distress in relation to medical procedures. Specifically, researchers have found that age is inversely related to the amount of child behavioral distress during immunizations administered to children during their first 2 years of life (Craig, McMahon, Morison, \& 
Zaskow, 1984) as well as various other acutely painful pediatric medical procedures (e.g., venipuncture in stratified age groups of children 3- to 17-years-old and finger-pricks in children 6 to 11 years of age; Goodenough et al., 1997; Bachanas \& Roberts, 1995, respectively). Extending these findings to acute painful medical procedures to children with cancer, studies have also found a significant, inverse relation between distress and chronological age during more invasive, painful medical procedures in oncology with children aged 8 months to 13 years (Hubert, Jay, Saltoun, \& Hayes, 1988; Jay, Elliot, Katz, \& Siegel, 1987; Katz et al., 1980).

Although more research is needed to uncover the reason for the commonly-observed inverse relation between age and distress reported by some authors, this relation may be due to age-related cognitive development and the use of different coping strategies by younger and older children (Altshuler \& Ruble, 1989; Band, 1990; Band \& Wiesz, 1988). Similarly, research suggests that the strategies younger children spontaneously use to cope with medically-related distress may be less effective at anxiety reduction as compared to those used by older children (Band \& Wiesz). Alternatively, researchers have investigated qualitative, as compared to quantitative, changes in infant pain behavior during routine immunizations as a function of age (Izard, Hembree, Dougherty, \& Spizzirri, 1983; Izard, Hembree, \& Huebner, 1987). Specifically, Izard and colleagues (1983) examined facial responses to immunization in a cross-sectional sample of infants aged 2 to 19 months and found that older infants displayed physical distress or pain behaviors for a smaller proportion of time, and displayed anger and blended emotional expressions (e.g., painsadness) for longer proportions. These findings were later replicated in a longitudinal 
sample of 2- to 7-month-old infants (Izard et al., 1983). Thus, distress expression may not change in quantity but rather in quality as a function of child age.

It is noteworthy, however, that several studies have countered findings linking age and distress by reporting no significant relation between age and distress (LeBaron \& Zelter, 1984; Weisz, McCabe, \& Dennig, 1994). Although the reasons for these discrepant results are unclear, they may be due in part to differences in the operationalization of distress across studies; some authors included more molar behaviors in the definitions of distress (e.g., scream; Schechter et al., 1991) whereas other authors examined more molecular behaviors (e.g., wince; LeBaron \& Zelter). It is possible that distress does not simply decrease with age, but rather may be expressed in more subtle ways as compared to the more obvious signs of distress observed during infancy.

Similar to age differences in procedural distress, there are somewhat mixed findings with regard to gender differences. Some studies have demonstrated that girls report (Hildegard \& LeBaron, 1982; Melamed \& Siegel, 1985, Wiesz et al., 1994) and exhibit (Hildegard \& LeBaron; Katz et al., 1980) more distress during painful medical procedures than boys. Although gender differences with regard to self-report of distress have been consistent across studies, several studies have failed to find gender differences with regard to overt distress behaviors (Hubert et al., 1988; Jacobsen, Manne, Gorfinkle, Shorr, Rapkin, $\&$ Redd, 1990; Wiesz et al.). The reason for this inconsistency is unclear; however, it may be related to differences in sample characteristics. For example, prior researchers (Cheng et al., 2003; McGrath, 1990) have conjectured that gender may interact with age in determining distress behaviors. That is, over time boys learn to adopt more stoic response to pain, while girls learn to express pain more affectively. Indeed, the studies in this literature 
that have not found gender differences in distress behavior included a more restricted age range (e.g., Hubert et al., Jacobsen et al.; Wiesz et al.; age ranges $3-11,3-10$, and 5 - 12, respectively) compared to the studies that reported significant gender differences (e.g., Hildegard \& LeBaron; Katz et al.; age ranges $6-19$ and $1-17$, respectively). It is also quite likely that differences in findings across studies may be due to methods of measuring distress. When distress has been self-reported, girls exhibit more distress than boys; however, when distress was measured using behavioral observations, gender differences were less likely to be observed. Thus, although there is some evidence that gender influences distress in response to immunization pain, this relation may vary as a function of chronological age or the differential tendencies between girls and boys in self-reporting distress.

In summary, results across studies have demonstrated age and gender differences in the distress behavior of children undergoing painful medical procedures. Specifically, older children typically show fewer signs of behavioral distress than younger ones and are more likely than younger children to employ more effective methods of coping with pain. Although some research has reported discrepant results in this regard, it is possible that these divergent results are due to differences in sample characteristics across studies. Gender differences in distress behaviors have also been fairly consistently reported. Although several researchers failed to find any gender differences (possibly related to differences in sample characteristics and methods of measurement across studies), when gender differences have been found, they suggest that girls exhibit more distress during medical procedures as compared to boys. 


\section{Child Temperament}

In addition to the literature examining age and gender as predictors of distress, researchers have recently begun to explore the role of child temperament in pediatric distress. Temperament is conceptualized as an individual's behavioral style or pattern of responding to external stimuli that presumably has biologic and genetic roots, begins while the child is in utero, and is relatively stable across time (Thomas \& Chess, 1977). Thomas and Chess conceptualized temperament as being comprised of nine categories (activity, rhythmicity, approach, adaptability, intensity, mood, persistence, distractibility, and threshold). From scores in each of these nine categories, children were grouped into three diagnostic clusters: (a) difficult (characterized by a tendency to withdraw, biological irregularity, high intensity, negative mood, and slow adaptability); (b) easy (rhythmic, mild, readily approachable, quick in adaptability and positive in mood); and (c) slow to warm up (low in activity, approach, and adaptability, negative in mood, mild and variable in rhythmicity; Thomas \& Chess).

There is some research to suggest that temperament influences children's nociception (i.e., "the neural transmission of information about stimuli that are causing tissue damage;" Sufka \& Price, 2002, pp. 278). Grunau, Whitefield, and Petrie (1994) found that temperament was significantly related to a general parental rating of pain sensitivity in toddlers who were of full birth weight and those who were heavier preterm infants (as compared to extremely low-birth-weight preterm infants). Using a composite assessment of temperament, Grunau and colleagues reported that more emotionally reactive toddlers were more sensitive to everyday pain, per parental report, than their less emotionally reactive peers. 
The role of temperament in children's everyday nociception has been extended to examine this relation in pediatric settings. Findings across studies have been fairly consistent in that difficult child temperament was positively associated with child distress during immunization (Schechter et al., 1991; Sweet et al., 1999) and venipuncture procedures (Lee \& White-Traut, 1996).

Schechter and colleagues examined child distress in the context of immunization procedures and temperament in a sample of 4 to 6 year olds. Temperament was assessed via the Behavioral Style Questionnaire (BSQ; McDevitt \& Carey, 1978), a measure that included the nine temperament dimensions originally conceptualized by Thomas \& Chess (1977). Results indicated that the cluster of difficult child temperament characteristics was significantly positively associated with the amount of child distress behavior. Moreover, the specific temperament dimension of adaptability bore the strongest significant, negative relation to child distress (Schechter et al.).

Other researchers have extended the examination of the relation between temperament and child distress among additional age groups and pediatric populations. Sweet and colleagues (1999) measured temperament using the Infant Characteristics Questionnaire (ICQ; Bates, Freeland, \& Lounsbury, 1979) and found difficult child temperament to be significantly positively associated with child distress in children undergoing 6- and 24month immunizations. Lee and White-Traut (1996) employed the BSQ (McDevitt \& Carey, 1978) to assess temperament in relation to child distress during a venipuncture procedure. Among their sample of 3- to 7-year-old children, results indicated that the difficult child temperament cluster was significantly positively associated with amount of child distress. 
Thus, it appears that difficult temperament is related to increased behavioral distress of toddlers and infants undergoing immunization and venipuncture procedures.

\section{Parent Health Care Attitudes}

Compared to the research linking historical and demographic variables and child temperament to pediatric distress, less attention has been paid in the literature to the relation between parent health care attitudes and pediatric distress. Only recently have researchers begun to explore the role of parents' healthcare attitudes in predicting pediatric distress. Bachanas and Roberts (1995) examined health care attitudes in mothers of children aged 6to 11-years undergoing a finger-prick blood test during an outpatient well-child medical examination. Maternal attitudes were assessed with a self-report, 24-item instrument designed to evaluate attitudes towards eight medically related topics (e.g., hospitals, shots, dentists, doctors). Results revealed a significant relation between child distress during finger-prick and maternal health care attitudes, with more positive attitudes related to less observable child distress behaviors.

Extending this literature to younger children, MacLaren and Cohen (2004) evaluated parental healthcare attitudes in parents of children undergoing pre-surgery venipuncture. The authors employed a 12-item measure to assess health care and injections attitudes in parents of 1- to 7-year-old children undergoing pre-surgery venipuncture. Results indicated that positive health care and injection attitudes were significantly inversely related to observable child distress, as well as parent and nurse reports of child distress during venipuncture. Moreover, a subscale of this measure that assessed attitudes specific to child procedural pain, bore a stronger inverse correlation with the aforementioned measures of child distress. The authors speculated that parent attitudes about children's needle pain might influence parent's 
and children's reactions to these events. Alternatively, it could be that their child's painful medical experiences have shaped parents' attitudes about needle procedures (MacLaren \& Cohen). Although these findings may extend to different pain stimuli, research is still needed to examine the relation between parent health care attitudes and child distress related to immunization procedures.

In addition to impacting child distress during pediatric procedures, research has begun to explore the role of parents' immunization attitudes upon adherence to immunization schedules (Prislin, Dyer, Blakely, \& Johnson, 1998). Prislin and colleagues examined attitudes and beliefs about immunizations in parents of children aged 2- to 24-months. Specifically, this assessment included questions regarding barriers to accessing immunizations, safety concerns about vaccines, knowledge about the medical contraindications of vaccines, and distrust of medical professionals. Adherence to immunization schedules was assessed via an up-to-date method wherein subjects were classified as up-to-date or not up-to-date based upon the number of immunization injections they had received by a particular age. Results indicated that more positive attitudes were related to better adherence to immunization schedules. It is important to note that, unlike the aforementioned studies that assessed attitudes in the context of pediatric distress, Prislin and colleagues' assessment of immunization attitudes did not specifically assess attitudes about child distress during immunizations. Parent attitudes towards their child's immunization distress may be another important component in understanding the relation between parent attitudes and adherence to immunization schedules. Thus, more research is needed to examine the relation between parent attitudes about pediatric procedural distress and adherence to immunization schedules. 


\section{Parent Psychopathology}

Except for prior exposure to procedural distress, age, gender, temperament, and parent health care attitudes, few additional variables have been investigated in predicting child distress during immunizations. This lack of empirical investigation is particularly evident with regard to parent psychopathology. Although there are currently no studies investigating other predictors of adherence to childhood immunization, it is reasonable to hypothesize that a parental characteristic like psychopathology may be related to distress at child immunizations. Because various psychopathologies may interfere with a parent's ability to appropriately prepare the child for a medical procedure or assist them during the procedure (e.g., promote coping behaviors in their child during the procedure; provide child with appropriate information regarding what to expect of the procedure), it is surprising that no studies have explored this relation. For example, a parent who is suffering from depression may be less inclined to actively promote coping throughout the procedure via appropriate interactions with their child.

In addition to impacting child distress during immunizations, it is also possible that parent psychopathology is related to adherence to immunization schedules. For example, a parent with an anxiety disorder may be more distressed upon seeing their infant in distress during immunizations as compared to a parent with less anxiety. In addition, it could be hypothesized that a particular form of anxiety, namely social anxiety, could render parents more sensitive to child immunization procedures in which they might perceive their inability to adequately comfort their child or manage their child's behavior as being negatively evaluated by medical staff. Alternatively, a parent suffering from depression may be less able to adhere to immunization schedules due to a decrease in activity level. Numerous other 
parental psychopathologies (e.g., thought disorders, avoidant personality disorder) may also put children at risk for missing immunizations. Despite the promise of exploring parental psychopathology and both magnitude of child distress during medical procedures and adherence to childhood immunization schedules, no research has been done to test these hypotheses.

\section{Adherence to Immunization Schedules}

One additional variable may be linked to the magnitude of distress children experience during medical procedures - the extent to which the child is familiar with immunization setting, most commonly the medical clinic. Recognizing that adherence to the recommended schedules of immunization results in regular clinic visits where immunizations typically occur, two competing hypotheses could be generated. On one hand, it could be hypothesized that the increased exposure to the clinic setting might result in a greater opportunity for a child's distress reactions to extinguish. On the other hand, due to the repeated pairings of clinic stimuli with injection pain, it could be hypothesized that the increased frequency of clinic visits associated with adherent children and their families would sensitize these distress reactions.

Research indicates that instances of nonadherence to schedules of immunization are related to parental concern about injection pain. Findings from a study by Meyerhoff and colleagues (2001) illustrate the extent to which parents of children aged 18 months to 7 years are concerned about minimizing their children's distress. The study attempted to quantify parent's negative reactions concerning immunization-related pain and distress by determining a dollar amount parents would be willing to pay to reduce emotional distress in their children during immunization procedures. Irrespective of socioeconomic status, parents 
indicated they were willing to pay an average amount of $\$ 57$ - $\$ 79$ to reduce their child's immunization distress (Meyerhoff et al.). Although research is lacking to explore the possible relation between parental concern about immunization pain and the associated distress of the child and nonadherence to immunization schedules, this concern may be related to the likelihood of parents bringing their child in for immunizations.

Immunization schedule adherence may be related to child distress during pediatric immunization. For example, it is possible that parents of infants who displayed greater distress behavior upon immunization may have experienced their child's immunization as a more distressing stimulus (e.g., child distress behaviors) than parents of infants who displayed relatively lesser amounts of distress behaviors. Moreover, in an attempt to avoid exposure to the distressing stimulus, these parents could miss future medical appointments, postpone immunization injections, and otherwise not adhere to the immunization schedule. Unfortunately, nonadherence of this type prevents the child from regular exposure to health care settings, possibly potentiating child distress during subsequent immunizations.

It could be hypothesized that immunization schedule adherence provides the child with exposure to medical setting stimuli on a regular basis, thus resulting in progressive reduction in child distress behaviors at subsequent pediatric immunizations. According to behavior analytic learning theory, stimuli associated with a distressing event (e.g., medical staff or clinic involved in the immunization procedure) could become conditioned to elicit distress (e.g., crying) through a previous history with an aversive stimulus (e.g., needle; Mowrer, 1947; for a recent review of this theory see McAllister \& McAllister, 1995). Children who display large amounts of distress during infant immunizations, but then experience multiple, less-distressing medical visits (via adherence to immunization schedules and pain behavior 
reduction related to child maturation effects), may have more positive experiences with subsequent immunizations; these positive experiences may serve to moderate their initial negative experience and result in less distress behaviors during subsequent immunizations. According to this perspective, immunization schedule adherence would be inversely related to child distress during pediatric immunization.

Alternatively, it could be hypothesized that repeated exposure to immunization injections conducted in clinic settings might result in increased childhood distress. According to the principles of respondent conditioning, neutral stimuli associated with the clinic setting could be conditioned to elicit conditioned emotional responses (i.e., child distress behaviors) with repeated pairings with aversive unconditioned stimuli (i.e., injection pain). Children from immunization-schedule-adherent families, then, would be predicted to exhibit greater distress due to the more frequent pairings of conditioned and unconditioned stimuli. Despite the conceptual foundation supporting these competing hypotheses, no studies have been conducted to explore the relation between adherence to immunization schedules and child distress during medical procedures.

\section{Statement of Purpose and Significance of the Study}

Despite the negative consequences associated with procedural distress in pediatric patients, as well as the large numbers of children who must undergo multiple immunizations, very little research has focused on predictors of pediatric distress during immunizations or other distressing medical procedures (e.g., Blount et al., 2003). In contrast to the relatively sparse and discrepant literature regarding predictors of pediatric distress, there are multiple pharmacological (e.g., local anesthetics, EMLA), and cognitive behavioral interventions (for review see Piira et al., 2002) that have demonstrated efficacy in alleviating pediatric 
immunization distress. However, the relative lack of research regarding predictors of pediatric distress limits clinical applications of these effective pain management interventions for individuals who need them the most. Although the effects of age, gender, previous exposure to distressing medical procedures, and child temperament upon measures of child distress during medical procedures have been reported in several studies, very few studies have examined the relation between parental health beliefs and child distress, and no studies have examined the relations between both parent psychopathology and adherence to immunization schedules and ratings of child distress during medical procedures.

The current study proposes to examine how each of these individual difference variables is related to child distress during immunizations at 12- to 18-months of age. In order to explore these possible relations, this study evaluated 50 children and one of their parents during a visit to a pediatric clinic during which an immunization was administered. Parents completed questionnaires measuring their child's temperament, child's prior medical experience, parent psychopathology, and parent's immunization beliefs. Behavioral observation data were gathered during the immunization procedure itself and coded for child distress behaviors. Finally, a medical chart review was conducted and attendance at a followup immunization appointment was recorded in order to gather information regarding immunization schedule adherence.

The primary aim of the study was to examine how child distress during $12-18$ month immunizations was related to the following variables: (a) Past medical distress, (b) Child age and gender, (c) Child temperament, (d) Parent health care beliefs, (e) Parent psychopathology, and (f) Immunization schedule adherence. It was hypothesized that level of past medical distress, difficult child temperament, and level of parent psychopathology 
would be significantly, positively correlated with child distress, and that adaptive health care beliefs would be significantly negatively correlated with child distress. Further, it was hypothesized that child gender would be significantly associated with distress, in that boys would exhibit significantly less distress than girls. Because such a restricted age range of children was used in this study, no relations were predicted between age and measures of child distress.

Although examination of the relations among each of these individual difference variables and measures of child distress during an immunization procedure is of interest, these variables are likely to not exert purely independent effects on measures of child distress. In this regard, a second aim of this study was to examine how designated individual difference variables interact to account for variance in child distress. Several specific interactions were examined based upon hypothesized relations. First, based upon the empirical evidence linking prior medical procedure-related distress to current child distress (Dalqhuist et al., 1986) and the hypothesized relation between prior medical procedurerelated distress and immunization schedule adherence, the interaction between prior medical procedure-related distress and immunization adherence upon measures of child distress was examined. It was hypothesized that distress would be the greatest among children with the most previous procedure-related distress and the poorest adherence to immunization schedules.

Second, based upon the empirical evidence linking parental health attitudes with both child distress (MacLaren \& Cohen, 2004) and immunization schedule adherence (Prislin et al., 1998), the interaction between parent's medical attitude and immunization adherence upon measures of child distress was examined. It was hypothesized that distress would be the 
greatest among children with parents with the least adaptive medical attitudes and the poorest adherence to immunization schedules.

Third, based upon the hypothesized association between parent psychopathology and child distress and immunization schedule adherence, the interaction between parent psychopathology and immunization adherence upon measures of child distress was examined. It was hypothesized that distress would be the greatest among children with parents with greater psychopathology and the poorest adherence to immunization schedules.

There was also a third aim of this investigation — to explore prospectively whether ratings of child distress influence subsequent adherence to a scheduled immunization appointment. If indeed previous procedure-related distress is associated with reduced adherence to schedules of immunization, it would logically follow that ratings of child distress observed during the clinic visit in this study might predict future attendance to clinic visits for purposes of immunization. In order to conduct this analysis, known risk factors for nonadherence to child immunization schedules were controlled, including family size, level of parental education, ethnicity, and family income. Larger family size, lower level of parent education, ethnic minority status, and lower family income have all repeatedly and significantly predicted lower levels of adherence to immunization schedules for children aged 2 years and under (e.g., Bobo, Bale, Thapa, \& Wassilack, 1993; Guyer et al., 1994; Institute of Medicine, 2000; Prislin, Dyer, Blakely, \& Johnson, 1998; Wood, et al., 1995). Controlling for these standard risk factors, it was hypothesized that measures of child distress would be associated with attendance at a future clinic visit for purposes of immunization.

This study is significant because knowledge of individual differences that are related to pain could allow health care professionals to better serve families in several ways, 
including aiding in the identification of families that are at risk for significant child distress and possible nonadherence to immunization schedules. Subsequently, intervention efforts (e.g., education interventions, pain management interventions) could be developed and tailored to best meet the needs of those at-risk individuals. Additionally, if pediatric distress and immunization schedule adherence are shown to be inversely related, then the use of pain management protocols that decrease pediatric distress may, in turn, lead to increased adherence to immunization schedules.

\section{Method}

\section{Participants}

Seventy children between the ages of 12 and 18 months and one of their parents were recruited to participate in this study from the Pediatric and Adolescent Group Practice Clinic at the Robert C. Byrd Health Sciences Center of West Virginia University. Eleven of these children and their parents met inclusion criteria for the current study but declined to participate. Parents cited a lack of desire to be videotaped $(n=6)$, insufficient fluency in the English language $(\mathrm{n}=2)$, a disinterest in the aims of the current research study $(\mathrm{n}=2)$, and a lack of time $(\mathrm{n}=1)$ as reasons for declining. Of the 59 children and parents who agreed to participate in the study, 9 ( 8 boys and 1 girl; 8 Caucasian and 1 Pakistani) were excluded from analyses due to missing data (i.e., child did not require any follow-up immunizations until 5 years of age). The final study sample was comprised of 50 children (24 males; 26 females) between the ages of 12 and 18 months $(M=14.0$ months; $\mathrm{SD}=2.58)$ receiving at least one immunization injection during their clinic visit and their accompanying first degree biological relative or adoptive parent. 


\section{Measures}

Patient Information Form (PIF). The PIF (Appendix A), designed for the purposes of this study, was utilized to gather descriptive information about participating families, including basic demographic variables and general medical information about the child. Modified Behavioral Pain Scale (MBPS) (Taddio, Nulman, Goldbach, Ipp, \& Koren, 1994). Child behavioral distress was assessed via the application of the MBPS coding system to videotaped recordings of the immunization injection procedure. The MBPS is a revised version of the Children's Hospital of Eastern Ontario Pain Scale (CHEOPS; McGrath, Johnson, Goodman, Schillinger, Dunn, \& Chapman, 1985), specifically for use with injection pain. The MBPS is a molar measure of pain that includes three behavioral subcodes indicative of procedural distress (i.e., facial expression, cry, and body movement of the torso, arms, and legs). The MBPS has demonstrated good reliability and validity (e.g., total MBPS score intraclass correlation coefficient $=.95$; Taddio, Nulman, Koren, Stevens, $\&$ Koren, 1995).

To provide a finer level of detail, coding in this study was divided into four phases: baseline (20 s until $10 \mathrm{~s}$ prior to injection), anticipatory (10 s prior to injection until injection), injection (injection until $10 \mathrm{~s}$ later), and recovery (20 s following the final injection until $10 \mathrm{~s}$ later). Thus, each child received an MBPS score ranging from 0 to 10 for each of the four phases with a total score ranging from 0 to 40 . As a means of facilitating comparisons across phases, an MBPS total score was derived by averaging scores across Facial Expression, Cry, and Movements. Therefore, the overall MBPS scores for each phase ranged from 0 (minimum distress) to 3.33 (maximum distress). 
A research assistant who was kept blind to study hypotheses was trained on the MBPS using videotapes from participants excluded from data analyses until $90 \%$ agreement with the primary investigator was obtained. In accord with the MBPS protocol, the coder assigned an anchored score of 0, 1, 2, or 3 for Facial Expression and Cry and a score of 0, 2, 3, or 4 for Movements (i.e., " 0 " is coded for both resting and usual activity movements) for each 10-s interval. For example, a score of 0 on Cry corresponded to "laughing or giggling" whereas a score of 3 indicated "full lunged cry or sobbing."

Observer agreement via Cohen's kappa $(\mathrm{k})$ was calculated based upon ratings made from a random set of $20 \%$ of the videotaped injection procedures. Cohen's kappa is a conservative statistic that corrects for chance agreement (Bakeman \& Gottman, 1987; Cohen, 1960). Kappa correlation coefficients were calculated for each of the total scores of the four procedural phase subcodes. Inter-rater agreement scores for the procedural phase total subcodes ranged from .84 to .92 , with a mean kappa of .87 . Individual subcode kappa scores were: (a) Total baseline period $=.92$, (b) Total anticipatory period $=.88$, (c) Total injection period $=.84$, (d) Total recovery period $=.85$, (e) Total facial expression $=.80$, (f) Total cry $=$ .93 , and $(\mathrm{g})$ Total movements $=.89$.

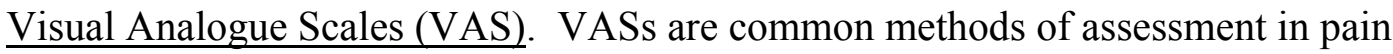
studies and have been shown to be valid (McGrath, 1990). For example, VASs have been shown to successfully quantify sensory intensity and affective aspects of laboratory-induced pain stimuli in a manner that was not significantly different as compared to verbal reports of pain (Duncan, Bushnell, \& Lavigne, 1989). One strength of using VAS measures is that values do not typically result in a clustering of scores that often occurs with categorical scales (Varni, Walco, \& Wilcox, 1990). All VASs employed $100 \mathrm{~mm}$ lines anchored with 
"Not distressed at all" and "Very distressed." In order to assess child distress during prior medical experiences, caregivers completed a VAS questionnaire (prior to the injection procedure) based upon similar measures used in the literature (Dalqhuist et al., 1986; Appendix B). Sample questions include: "How distressed were you during your child's first immunization procedure?" and "How distressed was your child during his/her first immunization procedure?" Responses to items regarding prior medical experience were summed in order to calculate a Prior Medical Experience Score, with higher scores indicating more child distress during prior medical procedures. Upon conclusion of the immunization injection procedure, caregivers and nurses independently completed VASs in response to the questions such as "How distressed were you during the procedure?" and "How distressed was the child during the procedure?" (See Appendix C).

Toddler Temperament Scale (TTS) (Fullard, McDevitt, \& Carey, 1984). This 97-item questionnaire was designed to assess the temperamental characteristics of children aged 1 to 3 years. Nine temperament dimensions or categories were assessed: activity level, regularity, approach-withdrawal, adaptability, intensity, mood, persistence, distractibility, and sensory threshold. Normative data is available for males and females from 12 to 36 months of age. Scores were grouped into five clusters according to the procedures outlined by Carey (1970). Three clusters consist of the "easy" child, the "difficult" child, and the "slow-to-warm-up" child (Carey). Children whose scores did not meet criteria for membership in the aforementioned categories were designated as "intermediate high" (toward the "difficult" group) and "intermediate low" (toward the "easy group") based upon the number of category scores that fell on the "difficult" side of the mean. For the purposes of the present investigation, the children whose scores placed them in either the "difficult" or "intermediate 
high" groups were operationalized as children of a difficult temperament. Both "easy" and "slow-to-warm-up" scores were coded as "non-difficult child." The TTS has demonstrated satisfactory temporal stability over a 1-month period of time, with median test-retest correlations ranging from .70 and .81 , respectively, for the nine category scales.

Health Care and Injection and Attitudes Questionnaire (HCIAQ). (MacLaren, McCourt, \& Cohen, 2004). The HCIAQ is a 12-item measure designed to measure the health care attitudes of parents of young children. Responses to statements indicative of health care attitudes (e.g., "I enjoy taking my child in for health care") are rated using a 5-point Likerttype scale with anchors "Strongly Agree," "Agree," Neutral," "Disagree," and "Strongly Disagree." Higher scores on this measure are indicative of more adaptive health care attitudes. Although other measures of health care attitudes exist (e.g., the Health Opinion Survey-Parent; Strube et al., 1991, and the Health Care Attitudes Questionnaire; Hackworth \& McMahon, 1991), the HCIAQ is the only measure that is sufficiently brief for feasible administration in a fast-paced applied setting such as a typical pediatric clinic. Further, research has indicated that this measure has predictive validity ( $r$ s range from -.27 to -.35 with child distress measures) as well as adequate internal consistency ( $\alpha=.69$; MacLaren et al.) in a study measuring child and parent distress during venipuncture procedures. In keeping with the aims of the current study, scores from a subset of items on the HCIAQ that focus solely on procedural pain were employed in analyses (MacLaren \& Cohen, 2004). This score, which consists of seven items that pertain to procedural anxiety and pain, has demonstrated superior psychometric properties when compared to those of the full-scale score ( $r$ s range from -.47 to -.52 with child distress measures; $\alpha=.75$; MacLaren $\&$ Cohen). Item responses included in analyses are comprised of the following seven items: (a) "The 
doctors/nurses know how to help when my child is feeling distressed," (b) "I dread taking my child in for immunizations or other medical care," (c) "Injections are distressing to my child,"(d) "Injections are painful to my child," (e) "I am able to help my child when he/she is distressed during a medical procedure," (f) "Children's immunizations are too distressing for children," and (g) "Children's immunizations are too distressing for parents."

Brief Symptom Inventory (BSI) (Derogatis, 1993). The BSI is a 53-item, brief psychological self-report symptom scale that was utilized to assess parent psychopathology. This instrument uses a five-point Likert-type response format and is used to evaluate psychological symptoms across nine domains: somatization, obsessive-compulsive, interpersonal sensitivity, depression, anxiety, hostility, phobic anxiety, paranoid ideation, and psychoticism. In addition to these subscales, overall ratings of psychopathology were obtained via the Global Severity Index, Positive Symptom Distress Index, and Positive Symptom Total. Normative data exist for numerous populations, including male and female adult non-psychiatric patient norms. The BSI has demonstrated adequate temporal stability over a period of 2 weeks (symptom dimensions range from $r=.68$ to .91 ), as well as good internal consistency ( $\alpha=.71$ to .85 ; Derogatis). For the purposes of this study, raw scores were used to calculate the Global Severity Index score by summing values for the nine symptom dimensions and dividing by the total number of responses.

Immunization schedule adherence. Several methods of assessing adherence to immunization schedules were employed in this study. The primary measure of adherence was the attendance outcome at a follow-up immunization appointment (i.e., attend, no-show, canceled) scheduled at the time of study enrollment. In addition, up-to-date immunization status, which is the most commonly employed method of assessing immunization status, was 
assessed dichotomously from a review of the child's medical chart, wherein children were classified as "up-to-date" or "not up-to-date" according to whether they had received each recommended immunization. According to this method, each child was categorized as being either up-to-date with regard to all recommended immunizations, or they were not up-to-date if they had missed one or more recommended immunization injections. Despite the prevalence of the up-to-date method of immunization schedule adherence, several drawbacks are present with this method. For example, this method does not calculate the number of late immunizations, nor does it differentiate between children who lack just one immunization as compared to those who lack all of the recommended immunizations.

In order to allow for a more in-depth assessment of immunization schedule adherence, the Immunization Delivery Effectiveness Score (IDEA; Glauber, 2003) was also employed to assess adherence. The IDEA is a new index of immunization status that assesses, on a continuous scale, the timeliness of administration of each vaccination with reference to recommended age intervals. Specifically, a vaccine-dose IDEA score for each child was calculated with regard to each recommended vaccination event. The actual age of the child at administration of each of these vaccinations, with reference to the recommended age of administration generates the vaccine-dose IDEA score. A child's composite IDEA score is obtained by averaging the vaccine-dose IDEA scores for each immunization for that child.

Although the vaccination schedule is revised annually, it has remained the same in content since January, 2001. Therefore, for the purpose of this study, adherence to immunization schedule measures was based upon the January, 2004 recommended childhood and adolescent immunization schedule for the United States (CDC, 2003). 
Reasons for nonadherence form. The Reasons for Nonadherence Form (Appendix D), designed for the purposes of this study, was utilized to gather descriptive information about participating families' reasons for not attending the child's follow-up immunization appointment.

\section{Instrumentation}

Immunizations of all participants were video recorded using High-8 digital cassette recording tapes and a Sony digital camcorder camera mounted on a tripod. For coding purposes, a playback unit was used that included a color display screen, date and time (reading to tenths of seconds) settings, as well as stop action and slow motion feedback settings.

\section{Experimental Environment}

Immunization procedures were carried out in small clinic exam rooms regularly used for both medical examinations as well as routine immunization procedures in the Pediatric and Adolescent Group Practice Clinic at the Robert C. Byrd Health Sciences Center of West Virginia University. Data were collected in actual exam rooms of a busy pediatric primary care clinic in order to optimize generalizability to typical pediatric clinic settings. The majority of injections (78\%) were administered by one of three full-time pediatric nursing staff members, while the remaining injections (18\%) were administered by supervised nursing students; due to nurse staffing difficulties, 2 injections (4\%) were administered by a substitute nurse who typically worked in another pediatric department within the Robert C. Byrd Health Sciences Center. The overwhelming majority of infants were placed supine on the lower edge of an exam table with legs dangling over the end of the table. In this position, the nurse immobilized the legs of the child while the caregiver was verbally instructed to 
restrain the child's arms. In a couple of cases $(n=2)$, the parent was instructed to hold the child on his or her lap during the immunization procedure.

\section{Procedure}

The research team was comprised of the principal investigator and four undergraduate research assistants who were trained to collect and code data for the present investigation. In order to minimize experimenter bias, the four research assistants who collected and coded data where kept blind to study hypotheses. One member of the team approached qualified children and parents while they were waiting to see a physician for an immunization visit. Parents received a description of the purpose of the study, participation requirements, as well as participant rights. An approved consent form was reviewed with the parent and any questions were answered before the parent was asked to sign. Consent and the Health Insurance Portability and Accountability Act (HIPAA) authorization to access medical records of the child by the parent was required for participation. Additionally, all parents were required to schedule a next immunization appointment at the time of study enrollment. At the time of enrollment, parents completed the following measures: PIF, BSI, TTS, VAS (parent version, prior injections), and the HCIAQ. Research assistants also conducted a medical chart review in order to gather retrospective data regarding number of child's medical visits over the past 6-months and time-elapsed since last medical visit.

Once the above measures were completed, a research assistant accompanied each family to a treatment room where the video camera was placed and cued to record the immunization injection procedure. A wide angle lens was used to record parent, child, and nurse behaviors. The research assistant began the video recording upon the family's entrance into the treatment room. Although the research assistant remained in the treatment room for 
the duration of the procedure, he or she remained silent and avoided any interaction with medical staff, child, or parent while in the treatment room. Video recording continued until the conclusion of immunization injection procedure. Following completion of the immunization procedure, the research assistant turned off the camera and provided the parent and nurse with the post-injection VAS forms to complete. Although research assistants were prepared to make appropriate referrals for any medically or psychologically related concerns or questions participants posed to researchers, no such referrals were required. Research assistants escorted families to the check-out desk, where a follow-up immunization appointment was scheduled to occur within the next 6 months. Parents were told that their attendance at this follow-up immunization appointment would be recorded. Parents were alerted that that any cancellation or otherwise non-attendance at this appointment would prompt a brief assessment survey to be sent to them via mail that would assess their reason(s) for non-attendance. These questionnaires were mailed to parents who did not attend their child's follow-up immunization appointment within 4 weeks of their scheduled follow-up appointment.

\section{Results}

\section{Preliminary Analyses}

All continuous variables were inspected for signs of skew, kurtosis, and outliers via visual inspection of histograms and box plots. Estimates of skewness and kurtosis were also calculated for each variable; there were no variables that exhibited significant skew ( $>3)$ or kurtosis (>10). Outliers were observed in the distributions of four primary variables: (a) BSI Global Severity Index, (b) HCIAQ procedural anxiety and pain subscale score, (c) prior medical experience score, and (d) MBPS injection phase score. In addition, outliers were 
identified in each of the eight subscale scores of the BSI. For each of these variables, comparisons between original means and mean scores using the five percent trimmed mean (i.e., the mean recalculated with the top and bottom five percent of cases removed) were conducted. The original and trimmed means were found to be nearly identical for all variables except those scores obtained from the BSI, indicating that the outlying scores were not significantly influencing the data. For the BSI subscale and global severity index scores, in which inclusion of the outliers resulted in a significant difference between the observed and trimmed means (BSI Global Severity Index), each outlying score was replaced by the next highest (but non-extreme) score in order to retain these participants in the distribution while reducing the influence of the extreme score.

Due to technical difficulties in gathering video data and participants not completing all measures, there was some missing data. In terms of children's distress, 44 children (88\%) were obstructed during videotaping (i.e., by a nurse or caregiver standing between the video camera and the child) during some portion of the procedure. Fourteen children (28\%) were obstructed during the baseline phase of the injection procedure, 7 children (14\%) during the pre-injection phase, 2 children (4\%) during the injection phase, and 9 children (18\%) during the recovery phase of the injection procedure. For coding each phase (baseline, pre-injection, injection, recovery), these data were considered missing for purposes of analysis and compensatory actions were not taken (e.g., inserting a mean value).

Missing data also existed for 2 families who were new to the area and did not have complete immunization records in the medical chart. For purposes of evaluating adherence to the recommended immunization schedule, data from these 2 families were not included for analyses examining the effect of immunization adherence upon child distress. Finally, 7 
caregivers (14\%) endorsed all items on the BSI with the same value (e.g., all responses were rated as "1"); because of the clear response bias displayed during completion of this questionnaire among these participants, these data were not used in analyses evaluating the relation between parental psychopathology and ratings of child distress.

In order to prepare categorical demographic variables for analyses (i.e., create groupings wherein each group contained at least 5 participants), several variables were regrouped. Specifically, marital status was grouped into three categories (i.e., married to biological parent or step-parent, divorced/never been married, living together unmarried), respondent and spouse education variables were ordered into four categories (i.e., high school, some college, bachelor's degree, post graduate degree), occupation was organized into three categories (i.e., white collar, skilled/unskilled laborer, unemployed/disabled/ student), and child race was classified in two categories (i.e., Caucasian and Non-Caucasian). Medical insurance status of the participating child was not considered as a demographic variable in analyses, as all respondents indicated that their children had health insurance at the time of study enrollment. Finally, a caregiver who identified himself as not the "usual" care provider who attended immunization visits accompanied one child enrolled in the study. Due to the questionable validity of caregiver-reported past medical distress for this participant, data for this child's past medical distress were deleted and were treated as missing data in analyses.

\section{Descriptive Statistics for Demographic Variables}

Descriptive statistics on demographic variables are depicted in Tables 1 and 2 and descriptive statistics on all dependent variables are depicted in Tables 3 through 7 . The study sample was comprised of 24 boys and 26 girls between the ages of 12 and 18 months $(\mathrm{M}=$ 
14.0 months; $\mathrm{SD}=2.58$ ) receiving at least one immunization injection during their clinic visit and their accompanying first degree biological relative or adoptive parent $(92 \%$ mothers; $8 \%$ fathers). The majority of the sample was comprised of Caucasian children (72\%) from intact families (56\%) from low to middle class socioeconomic backgrounds (see Table 1). Children participating in this study received from one to five intramuscular shots during the immunization procedure $(\mathrm{M}=2.42 ; \mathrm{SD}=.96)$. An analysis of MBPS subscale scores indicated that the movements subcode scores exhibited a range of $0-3(M=2.24, \mathrm{SD}$ $=.85)$; ranges for the cry $(\mathrm{M}=2.27, \mathrm{SD}=.64)$ and facial expressions subcodes $(\mathrm{M}=2.69 \mathrm{SD}$ $=.68)$ were each 0 to 3 as well.

In terms of recent past immunization appointment attendance, $30 \%$ of participants had not attended a medical appointment wherein immunization injections were administered over the past 6 months from study enrollment. Approximately half of the sample $(52 \%)$ attended an appointment wherein injections were administered once within the past 6 months and $16 \%$ of participants received shots on 2 occasions within 6 months prior to study enrollment.

Aim One: Individual Difference Variables and Measures of Child Distress during 12-18 Month Immunizations

The first aim of this study was examine whether the three measures of child distress during 12 - 18 month immunizations (i.e., parent-reported, nurse-reported, and behavioral ratings of distress) would be significantly related to the following individual difference variables: (a) Past medical distress, (b) Child age and gender, (c) Child temperament, (d) Parent health care beliefs, (e) Parent psychopathology, and (f) Immunization schedule adherence. Behavioral ratings during the injection were used as the primary indicator of 
behavioral distress in these analyses because the highest frequency of behavioral distress occurred during this phase of the procedure and the least amount of missing data was observed during this phase. In addition, comparable analyses were conducted using baseline, pre-injection, and recovery phases and in almost all cases, these analyses yielded no additional significant findings (beyond those obtained via analysis of the injection phase scores). In order to examine the hypothesized associations, a series of Pearson and point biserial correlation coefficients (conducted for continuous and categorical variables, respectively) were calculated between demographic variables and child distress variables (see Table 8) and between dependent variables and child distress variables (see Tables 9 - 12). Prior to examining the relations between individual difference variables of interest and measures of child distress, it was important to establish whether child distress was related to any other potentially confounding historical, medical, or family variables. In particular, it was important to determine whether recency of prior clinic visits or number of injections received during the current clinic visit were related to child distress. As seen in Table 8, no significant correlation coefficients were observed for any of these relations, nor were there any significant associations between family characteristics and measures of child distress. However, child race was related to both the parent report of child distress $(r=.33, p$ $<.01)$ and the nurse report of child distress $(r=.38, p<.01)$. In both cases, non-Caucasian children were rated as exhibiting more distress than Caucasian children. Finally, it is important to note that the behavioral observation measure of child distress was significantly correlated with both parent $(r=.41)$ and nurse $(r=.59)$ reports of child distress $(p<.01)$. Likewise, the parent and nurse reports of child distress bore a significant relation to one another $(r=.51, p<.01)$. 
Past Medical Distress. The ratings of past medical distress were not significantly correlated with the MBPS injection score $(r=.05)$ or the nurse report of child distress $(r$ $=.25, p>.05$ ). However, the past medical distress score was significantly positively correlated with parent report of child distress $(r=.30, p<.05)$.

Child Age and Gender. The correlation coefficients examining the relations between child age and the parent report $(r=-.05)$, and nurse report $(r=-.19)$ of child distress each failed to reach significance. However, the correlation coefficient examining the relation between the MBPS injection score and child age was statistically significant $(r=-.32, \mathrm{p}<$ .05 ); greater child distress was observed among younger children. The correlation coefficients testing the relations between child gender and the parent report $(r=-.08)$, and nurse report $(r=-.16)$ of child distress were each not significant. The correlation coefficient exploring the relation between the MBPS injection score and child gender was statistically significant $(r=-.30, p<.05)$, with boys exhibiting higher MBPS injection scores as compared to girls.

Child Temperament. The correlation coefficient examining the relation between the TTS diagnostic cluster score and parent report of child distress was statistically significant $(r$ $=-.46, p<.01)$, with the category of difficult child temperament being associated with greater parent reports of child distress. However, significant relations between the TTS diagnostic cluster score and the MBPS injection score $(r=-.17)$ and the nurse report of child distress $(r=-.23)$ were not observed.

Correlation coefficients examining the relations between the three measures of child distress and TTS subscale scores were also examined. Although the majority of these correlations were not significant (see Table 9), a few significant associations were observed. 
For purposes of interpreting these correlation coefficients, it is important to recall that higher subscale scores are associated with more difficult child temperament. The MBPS injection score and the TTS adaptability $(r=.32, p<.05), \operatorname{mood}(r=.35, p<.05)$, and threshold $(r=$ $.31, p<.05$ ) subscale scores were significantly correlated (i.e., greater positive mood, rythmicity, and adaptability associated with lower levels of observational distress). Additionally, the relations between the parent report of child distress and the TTS rhythmicity $(r=.30)$, approach $(r=.32)$, adaptability $(r=.38)$ subscale scores all reached significance $(p s<.05)$, again indicating that greater rhythmicity, approachability, and adaptability were associated with lower parent reports of child distress. The relation between the nurse report of child distress and the TTS mood subscale score similarly was significant $(r=.30, p<.05)$, indicating that a more positive mood was related to lower nurse reports of child distress.

Parent Health Care Attitudes. Correlational analyses testing the relations between the HCIAQ total score as well as procedural anxiety and pain subscale score and each of the three measures of child distress revealed significant inverse associations between both HCIAQ scores and parent report of child distress $(r \mathrm{~s}=-.42, p \mathrm{~s}<.01$; see Table 10$)$. The associations between the HCIAQ scores and both the MBPS injection score and the nurse report of child distress failed to reach significance.

Parent Psychopathology. Correlational analyses between the BSI global severity index (GSI) score and each of the three measures of child distress revealed a significant positive correlation with both the MBPS injection score $(r=.41, p<.01)$ and the parent report of distress $(r=.31, p<.05$; see Table 11). However, the relation between the GSI score and nurse report of child distress was not significant $(r=.30, p>.05)$. 
Correlation coefficients examining the relation between the three distress measures and BSI subscale scores were also examined. Associations between the MBPS injection score and the BSI somatization $(r=.32, p<.05)$, interpersonal sensitivity $(r=.37, p<.05)$, anxiety $(r=.33, p<.01)$, phobic anxiety $(r=.32, p<.05)$, paranoid ideation $(r=.39, p<$ $.05)$, and psychoticism $(r=.50, p<.01)$ subscale scores were significant. Relations between the parent rating of child distress and the BSI interpersonal sensitivity $(r=.31)$, and depression $(r=.38)$ subscale scores were significant $(p<.05)$. The association between the nurse report of child distress and the BSI anxiety $(r=.42, p<.01)$ and psychoticism $(r=.31$, $p<.05)$ subscale scores were each significant. In all cases, higher symptoms of psychopathology were associated with greater child distress during immunization.

Adherence to Immunization Schedules. Because all but 1 child was rated as being upto-date on immunizations during the current visit using the categorical approach, analyses were not conducted using this variable; rather, analyses of adherence to immunization were conducted only using the IDEA score for each child. As seen in Table 12, no significant relations between the IDEA score and each of the measures of child distress were observed.

\section{Relations between Child Distress and Individual Difference Variables. Several}

individual difference variables hypothesized to be related to measures of child distress during immunizations were found to be significantly associated with at least one of the measures of distress employed in this study using univariate correlational analyses. To explore which variables (or combination of variables) best explained variance in child distress during immunizations, three standard regression analyses were performed, one for each measure of child distress. Due to the exploratory nature of the research questions addressed by these analyses, standard multiple regressions were employed rather than stepwise or hierarchical 
approaches. In each analysis, child age, child gender, TTS diagnostic cluster, HCIAQ

subscale score, prior medical experience score, and BSI global severity index were entered as independent variables.

For the regression analysis designed to explain the variance in MBPS injection scores, the full model regression equation with all six independent variables was significant, $R^{2}=.34, F(6,34)=2.95, p=.02$. The BSI global severity index made the strongest, unique contribution in accounting for a statistically significant portion of the variance (beta $=.39, p$ $=.02)$, although child age also made a statistically significant contribution $($ beta $=-.33, p=$ $.03)$. No other variable contributed to the variance in MBPS injection scores (see Table 13).

The regression analysis designed to explain the variance in the parent report of child distress was also significant, $R^{2}=.37, F(5,36)=3.38, p=.01$. The TTS diagnostic cluster score made the only unique contribution in accounting for the variance in the parent report of child distress ( $b e t a=-.38, p=.02$; see Table 14).

Finally, the third regression analysis designed to explain the variance in the nurse report of child distress was not significant, $R^{2}=.19, F(5,36)=1.4, p>.05$. None of the independent variables contributed to the variance in the nurse report of child distress (see Table 15).

Aim Two: Interaction Variables among Individual Difference Variables and Measures of Child Distress during 12-18 Month Immunizations

To address the second aim of the study and examine how designated individual difference variables might interact to relate to child distress, interaction terms were created by multiplying the individual difference variables of interest and immunization adherence scores (i.e., IDEA scores). In creating these interaction terms, all variables were centered 
and multiplied by each corresponding, centered immunization adherence score to create an interaction term. The following interaction term variables were created in this manner: (a) Centered prior medical experience scores and centered immunization adherence scores (prior medical experience x IDEA), (b) Centered HCIAQ subscale scores and centered immunization adherence scores (HCIAQ x IDEA), and (c) Centered BSI global severity index scores and centered immunization adherence scores (BSI x IDEA). Finally, a set of three hierarchical regression analyses were conducted to determine the contribution of each of these interaction terms above and beyond the variance of each of the three child distress variables accounted for by main effect variables. For each regression analysis examining hypothesized interactions, variables were entered in two steps. The first step in the regression consisted of the two main effect variables while the second step was comprised of the designated interaction term.

Past Medical Distress and Adherence to Immunization Schedules. The first set of regression analyses was designed to examine whether children with histories of previous medical distress and had poorer adherence to schedules of immunization exhibited more distress during the current immunization visit. The first regression analysis in this set employed the MBPS injection score as the dependant variable with prior medical distress and IDEA scores entered as independent variables in the first step and the prior medical experience $\mathrm{x}$ IDEA scores entered in the second step. In this analysis, no significant effect for the independent variables entered in the first step of the equation was observed, $R^{2}=.002$, $F(2,42)=.05$ (see Table 16). However, the interaction term entered into the second step (i.e., prior medical experience $\mathrm{x}$ IDEA score) made a significant contribution in accounting for the variance in MBPS injection scores, $R^{2} \Delta=.14, F \Delta(1,41)=6.71, p=.03$. 
To examine the nature of this interaction, median splits were conducted on prior medical experience, categorizing participants into those with relatively positive medical experiences and those with relatively negative medical experience, and IDEA scores, categorizing participants into relatively low and high adherences groups. Mean comparisons among positive experience-high adherence, positive experience-low adherence, negative experience-high adherence, and negative experience-low adherence groups on child immunization distress, as measured by the MBPS, were conducted using Tukey tests at the .05 level of confidence. Although there were no statistically significant mean differences among MBPS scores for the four groups using this approach, the mean score on behavioral distress for the negative experience-low adherence group $(\underline{\mathrm{M}}=2.52, \underline{\mathrm{SD}}=.26)$ was slightly greater than the mean score on behavioral distress for the negative experience-high adherence group $(\underline{\mathrm{M}}=2.29, \underline{\mathrm{SD}}=.49)$, the positive experience-low adherence group $(\underline{\mathrm{M}}=2.29, \underline{\mathrm{SD}}=$ $.56)$, and the positive experience-high adherence group $(\underline{\mathrm{M}}=2.38, \underline{\mathrm{SD}}=.44)$. See Figure 1 for a visual representation of the nature of this interaction.

A second, similar regression analysis was conducted using parent report of child distress as the dependant variable with the independent variables (i.e., prior medical experience, IDEA, prior medical experience x IDEA) entered in the manner described previously. There was no significant effect for the independent variables entered in the first step of the equation, $R^{2}=.10, F(2,44)=2.31$, nor was there any significant contribution of the interaction term entered in the second step of the equation accounting for the variance in the parent report of child distress score, $R^{2} \Delta=.00, F \Delta(1,43)=.006$ (see Table 17).

Finally, the third regression analysis in this set utilized the nurse report of child distress as the dependant variable with the independent variables (i.e., prior medical 
experience scores, IDEA scores, prior medical experience x IDEA scores) entered as described previously. There was no significant effect for the independent variables entered in the first step of the equation, $R^{2}=.06, F(2,44)=1.55$, nor was the interaction term significant in accounting for variance of the nurse report of child distress scores in the second step, $R^{2} \Delta=.02, F \Delta(1,43)=1.02($ see Table 18$)$.

Parent Health Care Attitudes and Adherence to Immunization Schedules. The next set of regression analyses was designed to examine whether parent's health attitudes regarding immunization and poorer adherence to schedules of immunization resulted in more child distress during the current immunization visit. The first regression analysis in this set employed the MBPS injection score as the dependant variable with the HCIAQ (procedural pain subscale score) and IDEA scores entered as independent variables in the first step and the HCIAQ x IDEA scores entered in the second step. There was no significant effect for the independent variables entered in the first step of the equation, $R^{2}=.03, F(2,42)=.05$ (see Table 19). However, the interaction term entered into the second step made a statistically significant contribution, above and beyond that accounted for by the HCIAQ and IDEA scores individually, in accounting for variance in the MBPS injection scores, $R^{2} \Delta=.11, F \Delta$ $(1,41)=5.37, p=.02$. Figure 1 provides a visual illustration of the nature of this interaction.

To examine the nature of this interaction, median splits were conducted on HCIAQ subscale scores, categorizing participants into those with relatively adaptive health care attitudes and those with relatively maladaptive health care attitudes, and IDEA scores, categorizing participants into relatively low and high adherences groups. Mean comparisons among adaptive attitude-high adherence, adaptive attitude-low adherence, maladaptive attitude-high adherence, and maladaptive attitude-low adherence groups on child 
immunization distress, as measured by the MBPS, were conducted using Tukey tests at the .05 level of confidence. The mean score on behavioral distress for the maladaptive attitudelow adherence group $(\underline{\mathrm{M}}=2.70, \underline{\mathrm{SD}}=.25)$ was significantly greater than the mean score on behavioral distress for the maladaptive attitude-high adherence group $(\underline{\mathrm{M}}=2.10, \underline{\mathrm{SD}}=.57)$ as well as the adaptive attitude-low adherence group $(\underline{\mathrm{M}}=2.19, \underline{\mathrm{SD}}=.43)$. The mean score on behavioral distress for the adaptive attitude-high adherence group $(\underline{\mathrm{M}}=2.51, \underline{\mathrm{SD}}=.44)$ did not differ significantly from any of the groups. See Figure 2 for a visual representation of the nature of this interaction.

A second, similar regression analysis employed the parent report of child distress as the dependant variable with the independent variables (i.e., HCIAQ scores, IDEA scores, HCIAQ x IDEA scores) entered in the manner described previously. There was a statistically significant effect for the independent variables entered in the first step of the equation, $R^{2}=.19, F(2,44)=5.09, p=.01$, with the HCIAQ scores being significantly related to child distress (see Table 20). However, the interaction term entered in the second step failed to account for any unique variance in the parent report of child distress above and beyond that accounted for by the HCIAQ and IDEA scores individually, $R^{2} \Delta=.02, F \Delta(1$, $43)=3.7$.

Finally, the third regression analysis in this set utilized the nurse report of child distress as the dependant variable with the independent variables (i.e., HCIAQ scores, IDEA scores, HCIAQ x IDEA scores) entered as described previously. There was no statistically significant effect for the independent variables entered in the first step of the equation, $R^{2}=$ $.02, F(2,44)=.52$, nor did the interaction term contribute to explaining any unique variance in the second step, $R^{2} \Delta=.04, F \Delta(1,43)=1.92($ see Table 21$)$. 
Parent Psychopathology and Adherence to Immunization Schedules. The final set of regression analyses was designed to examine whether degree of parent psychopathology and poorer adherence to schedules of immunization resulted in more child distress during the current immunization visit. The first regression analysis in this set employed the MBPS injection score as the dependant variable with the BSI global severity index and IDEA scores entered as independent variables in the first step and the BSI-GSI $x$ IDEA interaction term entered in the second step. There was a significant effect for the independent variables entered in the first step of the equation, $R^{2}=.19, F(2,36)=4.09, p=.03$, with the BSI-GSI being a significant independent variable in step one (see Table 22). The interaction term entered into the second step failed to account for any significant variance in MBPS injection scores, $R^{2} \Delta=.02, F \Delta(1,35)=1.05$.

A second, similar regression analysis employed the parent report of child distress as the dependant variable with the independent variables (i.e., BSI global severity index scores, IDEA scores, BSI x IDEA scores) entered in the manner described previously. There was a significant effect for the independent variables entered in the first step of the equation, $R^{2}=$ $.15, F(2,38)=3.22, p=.05$, with the BSI-GSI being a significant independent variable in step one (see Table 23). However, the interaction term entered into the second step failed to contribute significantly to the explanation of variance in the parent report of child distress scores, $R^{2} \Delta=.001, F \Delta(1,37)=.02$.

Finally, the third regression analysis in this set utilized the nurse report of child distress as the dependant variable with the independent variables (i.e., BSI global severity index scores, IDEA scores, BSI x IDEA scores) entered as described previously. There was no significant effect for the independent variables entered in the first step of the equation, $R^{2}$ 
$=.12, F(2,38)=2.5$ (see Table 24). The interaction term entered into the second step (i.e., the BSI x IDEA scores) failed to relate significantly to the nurse report of child distress scores, $R^{2} \Delta=.01, F \Delta(1,37)=.40$.

Aim Three: Measures of Child Distress during 12-18 Month Immunizations and Attendance at Future Immunization Appointment

The final aim of this study examined whether the extent of child distress observed during the current clinic visit influenced whether the parent and child kept the next scheduled clinic visit for purposes of immunization. Twenty-five children and their parents kept their follow-up visit and the remaining 25 children and parents did not attend the follow-up immunization appointment approximately 6 months after study enrollment. Data regarding reasons for nonattendance was obtained from only 7 participants who completed and returned the Reasons for Nonattendance Form. The remaining 18 participants who failed to keep the appointment also failed to return the Reasons for Nonattendance Form. Among those who returned the form, reasons endorsed for nonattendance at follow up visit included family member illness $(n=4)$, scheduling conflict $(n=2)$, and a family emergency $(n=1)$.

A set of three hierarchical logistic regression analyses were conducted to assess the relation between child distress and clinic visit attendance, controlling for several variables known to influence adherence: number of people in the home, annual household income level, parent education levels, ethnicity, and travel time to the medical facility. Examination of the univariate correlations between these demographic variables and attendance at the follow-up clinic visit revealed that annual family income $(r=.32, p<.05)$ was the only variable the bore a statistically significant relation to attendance at the follow-up clinic visit; all other correlations were not significant (see Table 25). Each logistic regression utilized the 
attendance at the follow-up clinic visit as the dependant variable. Number of people in the home, annual household income level, parent education levels, child race, and travel time to the medical facility, were entered as control variables in the first step; the designated measure of child distress (e.g., the MBPS injection score) was entered in the second step of each analysis.

The first logistic regression analysis examined whether child distress, as measured by MBPS injection scores, predicted attendance at the follow-up clinic visit, above and beyond any predictability in follow-up attendance accounted for by the control variables. None of the independent control variables entered into step one made a statistically significant contribution to the prediction of variance. The overall model summary for step one control variables was not significant as well, Nalgerke $R^{2}=.28, x^{2}(6,36)=8.33$. In step two, adding the MBPS injection scores to the regression equation accounted for a $.01 \%$ increment in the variance in follow-up attendance; the odds ratio suggested a one-unit increase in MBPS injection scores decreased the odds of attendance at the follow-up visit by $46 \%$ (odds ratio $=1.46)$. However, the full model regression equation with all seven predictors was not significant, $R^{2}=.29, x^{2}(7,36)=8.54($ See Table 26$)$.

The second regression analysis tested whether parent report of child distress score predicted attendance at the follow-up clinic visit, above and beyond any predictability in follow-up attendance accounted for by the control variables. All independent variables entered into step one failed to make a statistically significant contribution to the prediction of variance in the dependant variable. In step two, adding the parent report of child distress score accounted for a $.01 \%$ increment in the variance in follow-up attendance; the odds ratio suggested a one-unit increase in parent rating of child distress increased the odds of 
attendance at the follow-up visit by $8 \%($ odds ratio $=.92)$, also not a significant finding, $R^{2}=$ $.23, x^{2}(7,36)=7.06($ See Table 27$)$.

The final regression analysis examined whether nurse report of child distress predicted attendance at the follow-up clinic visit, above and beyond any predictability in follow-up attendance accounted for by the control variables. All independent variables entered into the first step of the equation failed to make a statistically significant contribution to the prediction of variance in the dependant variable. In step two, adding the parent report of child distress score accounted for a $.01 \%$ increment in the variance in follow-up attendance; the odds ratio suggested a one-unit increase in nurse rating of child distress increased the odds of attendance at the follow-up visit by $4 \%$ (odds ratio $=.96)$. The full model regression equation with seven predictors was not significant, $R^{2}=.29, x^{2}(7,36)=$ 8.54 (See Table 28).

\section{Discussion}

The primary purpose of the current investigation was to examine the relation of child distress during 12 - 18 month immunizations with the following variables: (a) Past medical distress, (b) Child age and gender, (c) Child temperament, (d) Parent health care beliefs, (e) Parent psychopathology, and (f) Immunization schedule adherence. This study involved children aged 12 - 18 months who were primarily Caucasian. Family and child variables were assessed via questionnaires and medical chart review, and a behavioral observation coding system was applied to videotaped recordings of the immunization procedure in order to assess child behavioral distress. Specific research questions examined included investigating (a) the relation between individual difference variables and measures of child distress during a 12 - 18 month immunization, (b) whether certain variables interacted with 
measures of immunization adherence in relating to child distress during a 12 - 18 month immunization, and (c) the relation between measures of child distress during a 12 - 18 month immunization and attendance at a future immunization appointment. A discussion of the current study's findings with reference to the literature, support and non-support found for study hypotheses, as well as implications for future directions for research and clinical applications follows.

Relation between past medical distress and distress during 12 - 18 month immunizations

Providing mixed support of hypotheses, results of the current study revealed that the quality of children's prior medical experience was significantly related to the parent report of child immunization distress, but was not significantly related to the direct observation or nurse report of child immunization distress. These findings contradict prior literature that has demonstrated a clear relation between the behavioral observation of child medical distress and the quality of children's prior medical experiences (i.e., Dalqhuist et al., 1986; Lumley, Melamed, \& Abeles, 1993). Although behavioral observations and nurse's reports of children's distress were not significantly associated with prior medical experience in the current study, parent report of child distress was related to prior medical experience. Nurse and parent reports of child distress have not been previously examined in relation to prior medical experience. There are several possible explanations for these partially unexpected results.

First, it is important to note that the current study is the first to investigate the impact of the quality of prior medical experience on present medical distress in children aged $12-18$ months. Prior studies linking the quality of past medical experiences to current procedural distress included both older children and larger age ranges (i.e., 3- to 12-year-olds, and 4- to 
7- year-olds; Dahlquist et al., 1986 and Lumley, Melamed, \& Abeles, 1993, respectively). It could be that, due to their younger age, children in the current sample had insufficient experience in medical settings to warrant development of significant distress reactions during 12 - 18 month immunizations, as assessed via direct observation and nurse report. Similarly, it has been hypothesized that the quality of prior medical experience influences subsequent procedural distress by influencing children's coping responses (adaptive or otherwise) to medical procedures. If this is so, it is quite likely that $12-18$ month old children have not formulated the types of coping strategies in response to prior medical procedures that older children would have acquired for use in later immunization visits.

An additional explanation of the discrepant findings between the current study and previous work focuses on the nature of the medical procedures employed to obtain measures of child distress. This is the first study to investigate the relation between quality of prior medical experience and child distress during an immunization procedure. The prior literature linking the quality of prior medical distress to current medical distress examined children undergoing throat swabs and anesthesia induction prior to elective ear, nose, or throat surgery (Dahlquist et al., 1986; Lumley, Melamed, \& Abeles, 1993, respectively). Throat swabs as well as anesthesia induction each involve little to no actual tissue damage and likely elicit less actual pain in children than the intramuscular injections used in this study. It is possible that in the context of more intense painful stimuli and thus, greater pain perception, the impact of prior medical experiences may be less predictive of child procedural distress behavior. The authors of both aforementioned studies reported overall low rates of procedure-related behavioral distress in their samples (Dahlquist; Lumley et al.). These findings stand in contrast to the moderate to high levels of behavioral distress documented in 
the current investigation and suggest that distress behaviors may occur at elevated rates for most children regardless of the quality of their previous interactions with medical clinics and medical professionals. Although the prior literature reported levels of child distress via use of a questionnaire similar in content to that used in the present investigation (i.e., Dalquist et al.), the published study used a different metric (Likert scale) and population (three to 12year-olds), making direct comparisons of mean scores difficult. Finally, the lack of significant findings relating prior medical distress with child distress during BMA (Katz et al., 1980) supports the interpretation that prior medical distress is less related to current distress in the context of more intense nociception.

In contrast to the lack of observed relations between behavioral and nurse-reported indicators of distress and prior medical distress, the relation between parent report of child immunization distress and prior medical distress was significant, supporting study hypotheses. However, it may be that the relation between parent report of child distress and prior medical distress, as documented in the current sample, was influenced by respondent bias. That is, parents who perceived their child's past medical experiences to be very negative may have been more likely to view their child's distress during the immunization procedure observed in this study in a negative light, thereby influencing responding on the parent report of child distress questionnaire. Thus, negative parent perceptions of prior medical experiences influenced parent behaviors during subsequent pediatric procedures, resulting in increased perceptions of their child's distress during the 12 - 18 month immunization procedure. It is also possible that the similar format of the questions assessing both parent report of distress and past medical distress may have led parents to respond consistently to both questionnaires that were administered typically within 1 hour of one 
another. Response sets based upon completing visual analog scales may also explain the greater congruence between parent ratings of distress and past medical distress than those observed between behavioral or nurse ratings of distress and past medical distress.

\section{Relation between child age and distress during 12 - 18 month immunizations}

Consistent with hypotheses, there was a significant, inverse relation found between child age and behavioral observation of child distress. This finding is consistent with previous empirical work (e.g., Bachanas \& Roberts, 1985; Goodenough et al., 1997), including investigations of immunization distress (Craig, McMahon, Morison, \& Zaskow, 1984) that have reported significant inverse relations among child distress and age. Moreover, analyses accounting for the relative relations contributions of the individual difference variables related to child distress in this study revealed that child age made a unique, significant contribution in accounting for the variance in child observational distress.

However, the relation between child age and distress was not replicated across the other child distress variables (i.e., parent and nurse report). It is pertinent to note that this is not the first instance in which a study failed to document age differences in child procedural distress across either multiple measures or a singular measure of child distress (e.g., Cohen, 2002, Weisz, McCabe, \& Dennig, 1994). Moreover, there are currently no published data demonstrating a relation between age and child procedural distress in the age group included in the current study. It is possible that the age range of the current sample was too constricted to permit detection of consistent relations between child age and distress.

Some have hypothesized that age differences in distress are qualitative rather than quantitative (e.g., Izard, Hembree, \& Huebner, 1987), with distress behaviors changing from more readily observed overt behaviors (e.g., screaming), to those of a more subtle nature 
(e.g., grimace) with age. In this manner, differences in child distress measures (e.g., molar vs. molecular behavioral distress measures) could reveal inconsistent results regarding the relation between child age and measures of child distress. That is, discrepant findings in age effects on distress, across measures of distress, may be due to differences in how child distress was operationalized across outcome measures. Thus, age differences in distress may have been too subtle or diffuse to detect via the parent and nurse report measures, but may only have emerged when a finer-grained analysis was applied via the behavioral coding system used to assess child distress via direct observation.

Alternatively, it is possible that the memory of previous immunizations among the current sample played a role in the relation documented between age and behavioral observation of immunization distress. For example, it may be that the younger children in the current sample displayed higher levels of immunization distress because the time period between the observed immunization procedure and the most recent, past immunization procedure was of a shorter interval, as compared to children who were older, and thus may have had a longer interval between the current immunization procedure and the last most recent one. More salient or readily retrieved memories of prior immunization procedures may set the occasion for increased distress in response to a 12 to 18 month immunization procedure.

Relation between child gender and distress during 12 - 18 month immunizations

A statistically significant relation was found between the behavioral observation of child distress and child gender, with boys in the current sample exhibiting greater amounts of behavioral distress, than girls, during a 12 - 18 month immunization procedure. Although a relation between child gender and child distress was hypothesized (i.e., girls would exhibit 
greater immunization distress than boys), the direction of the observed association was opposite of the hypothesized relation. Moreover, no relation was found between child gender and either nurse or parent report of child distress. Although these unexpected and nonsignificant findings disagree with current study hypotheses and with much of the prior literature suggesting that girls exhibit more distress during painful procedures, the literature remains mixed in support of this relation.

The finding linking male gender with increased direct observation of distress in the current sample is not without precedence (i.e., Grunau \& Craig, 1987, Meagel, Houser \& Gleaves, 1998). For example, in a distraction intervention study of children undergoing immunization procedures at 3 to 6 years of age, boys were found to exhibit greater overt distress responses during an immunization procedure than girls (Meagel et al.); this finding was documented across both control and experimental study groups. Moreover, Grunau and Craig conducted a study of neonatal gender differences in pain expression in response to a heel lance. Results of this study revealed that latencies of facial pain activity and time to cry were shorter in males as compared to females. Several studies have failed to find gender differences in pediatric distress (Cohen, 2002; Hubert et al., 1988; Jacobsen, Manne, Gorfinkle, Shorr, Rapkin, \& Redd, 1990). In this regard, the lack of significant findings with respect to the relation between gender and parent and nurse reports of child distress is consistent with this literature.

It is possible that the relation between males and increased behavioral distress in response to immunization observed in this study is unique to $12-18$ month old children. The majority of prior studies in this literature that have not found gender differences in distress behavior used relatively restricted age ranges (e.g., Cohen, 2002; Hubert et al., Jacobsen et 
al.; Wiesz et al.; age ranges 12 months and younger, $3-11,3-10$, and 5-12, respectively). It may be that the relation between gender and child distress are significant only when children across larger age ranges are included. It is possible that boys exhibit more distress as infants, but tend to show less and less distress as they are socialized according to prevailing gender norms. Gender identities and roles are very early in their formative stages during the 12 - 18 month period employed in the current study, and the social expectation that "big boys don't cry" is likely to play lesser of a role among the $12-18$ month olds used in this study than it would for older boys and girls. Clearly, the inconsistency in results regarding the relation between gender and pediatric distress both in the current as well as previous studies warrants a more systematic exploration in future studies across broader ranges of ages.

Finally, variables that were not directly assessed in the current study might be responsible for the unexpected relation documented between gender and distress. For example, a child's behavioral state (e.g., crying, asleep) immediately prior to a medical procedure is known to be related to child distress (Grunau \& Craig, 1987). In addition, it is possible that the increased levels of distress in males in the current sample, as compared to the girls, was due to increased anger expression; the behavioral observation coding system employed in the present study was not designed to detect affect. However, since these parameters were not assessed in the current study, the possibilities of differential preimmunization visit behavioral states and/or differential expression of affect among boys and girls are purely speculative.

Relation between child temperament and distress during 12 - 18 month immunizations

Mixed support was also found for the hypothesis that children with a difficult temperament would display more distress behaviors at a $12-18$ month immunizations than 
children with easy or slow-to-warm temperaments. Although difficult child temperament was not related to child distress measured by direct observation or nurse report, parent report of distress was associated with difficult child temperament. Analyses of temperament subscales also were linked to several indicators of child immunization distress. Specifically, greater levels of adaptability and threshold, as well as more positive mood, were all significantly associated with less observation of behavioral distress during the injection phase of the immunization procedure. In addition, greater levels of rhythmicity, approach, and adaptability were associated with lower parent reports of child distress during the immunization procedure and greater child mood was associated with less nurse report of child immunization distress. Thus, in the study sample, the pattern of distinct temperament dimensions related to each measure of child distress differed based upon the method of measuring distress. These findings are similar to those of previous empirical work. For example, Schecter et al. (1991) found a significant relation between difficult child temperament and observational immunization distress, with the adaptability subscale of the TTS best relating to immunization distress. In contrast, Lee and White-Traut (1996) found a significant relation between the threshold dimension of temperament, in addition to the difficult child diagnostic cluster, and child distress (as measured via behavioral observation and self-report of distress) during a venipuncture. In these cases, data obtained from the present investigation were comparable to previous empirical work, and TTS scores were comparable to those obtained in the initial TTS validation sample (Fullard, McDevitt, \& Carey, 1984) as well as in the literature on immunization pain (Schecter et al., 1991). Relation between parent health care attitudes and distress during 12 - 18 month immunizations 
Consistent with study hypotheses, parent health care and injection attitudes were significantly associated with the parent report of child immunization distress such that as adaptive health care attitudes increased, ratings of child distress decreased. As such, more adaptive parental attitude toward child immunizations was significantly related to less child distress during immunizations (e.g., Bachanas \& Roberts, 1995; MacLaren, McCourt \& Cohen, 2004). Although this finding is correlational, and causal interpretation not possible, there are a few contrasting explanations of how parental immunization attitudes and child distress during immunization are linked. On the one hand, it could be argued that parental attitudes about immunization distress influence their own reactions to these events, which in turn influence children's distress levels. The more negative attitude regarding immunization leads to negative mood and behavior of the parent and consequently the child. Alternatively, it is possible that higher levels of child immunization distress have shaped subsequent parental attitudes about immunizations.

It is important to note that the relation between parental attitude regarding immunization and child distress was not observed for behavioral and nurse report measures of child distress during immunization. There are several viable explanations for these mixed findings.

First, the discrepancy between the findings of the current study and those of prior studies may be due to differences in sample characteristics. For example, the relation between observational distress and parent health care attitudes has been documented previously in a sample of 6 to 8 year olds undergoing finger-pricks (Bachanas \& Roberts, 1995 ) as well as 1 to 7 year olds undergoing pre-surgery venipuncture (MacLaren \& Cohen, 2004). It is possible that the relation between parent health care attitudes and observational 
measures of child distress is less apparent in younger children or groups of children with a limited age range, like that used in the current sample. Similarly, it is possible that, while parent perceptions of child distress are related to parent health care attitudes in a younger sample of children, over time parent perceptions of child distress shape the behaviors of children in such a way that child distress is more readily observed via other measures of distress (e.g., direct observation, nurse report). For example, parents with more maladaptive health care attitudes may be more inclined to exhibit certain behaviors (e.g., behaviors displaying anxiety or mistrust of medical professionals) that are modeled for their child. Child acquisition of distress behaviors via modeling may not be readily detectable until the child is older than the ages examined in the current sample.

In must be noted that no prior studies have examined parent health care attitudes and child distress during an immunization procedure. Intramuscular injections result in more tissue damage and are more painful than finger prick and venipuncture procedures that have been examined in previous studies linking parent health care attitudes with child distress. Despite these study differences, immunization beliefs scores in the present study were nearly identical to those of other published data (MacLaren, McCourt, \& Cohen, 2004). It is possible that pain behaviors as measured via observational and nurse report assessments during intramuscular injections are not significantly influenced by parent health care attitudes because less learning (i.e., via parent modeling of maladaptive behaviors during a medical procedure) is possible in the context of a more salient pain stimuli, as compared to the conditioning that may take place in procedures that offer less intense pain stimuli. In other words, it would be difficult for a child to observe a parent's reaction during immunization when the child is screaming intensely with his or her eyes shut tight. Finally, it is possible 
that parents in this sample responded to questions about their child's immunization distress in a biased manner, with responding influenced by their own health care attitudes.

\section{Relation between parent psychopathology and distress during 12 - 18 month immunizations}

Support was found for the hypothesis that parent psychopathology would be related to child distress during a 12 - 18 month immunization procedure. Specifically, observational and parent report measures of child immunization distress revealed that parents who endorsed more symptoms of psychopathology had children who displayed greater levels of child immunization distress. Moreover, an analysis of the relative contributions of the individual difference variables linked with child distress revealed that parent psychopathology best explained variance in the observation of child distress during a 12 - 18 month immunization procedure, as compared to all variables examined in this study. However, these relations were not observed via nurse report of child distress.

A more in depth analysis of the relation between parent psychopathology and child immunization distress revealed several distinct psychopathology subscales that were related to immunization distress. For example, greater levels of anxiety were associated with increased distress across each of the three measures of child distress during immunization (i.e., observational, parent report, and nurse report).

These findings contribute greatly to the literature, as there were currently no previously published studies examining the relation between child distress during immunization and parent psychopathology. Furthermore, results indicated that a specific type of symptom (e.g., anxiety subscale) better related to child distress, across all three outcome measures, than the overall index of psychopathology or other subscale measures. Levels of parent psychopathology may interfere with a parent's ability to appropriately 
prepare their child for a medical procedure or assist them during the procedure (e.g., facilitating use of adaptive coping behaviors during the procedure; providing developmentally appropriate information regarding medical procedure). It also could be that parents who endorse more symptoms of anxiety also display more anxious behaviors during a 12 - 18 month immunization procedure, thereby inadvertently modeling distress behaviors to their child. However, it is important to note that, without further identification of characteristics of psychopathology best related to child distress, interpretation of specific mechanisms of psychopathology (e.g., parent modeling of anxiety or distress behavior to child during procedure) resulting in child distress remains unclear.

Similarly, it is possible that the parents who endorsed higher levels of anxiety also have children who display more anxious behaviors. Prior literature indicates that affective disorders in general, and anxiety disorders in specific, do bear a genetic component. For example, the rates of anxiety disorders among children of anxiety disordered parents is increased (e.g., Fyer, Mannuzza, Chapman, Martin, \& Klein, 1995; Maier, Lichtermann, Oehrlein, \& Franke, 1993; Mendlewicz, Papadimitiou, \& Wilmotte, 1993; Stein, et al., 1998), as compared to children without a family history of anxiety disorders. It may be that the parents in the current sample who endorsed higher levels of anxiety were more likely to have children who also displayed higher levels of anxious behaviors. The methods employed to assess immunization distress in the current investigation may have tapped children's more generalized, anxious responding to an unfamiliar situation, as well as immunization related distress responding.

Finally, it is important to note that the levels of psychopathology documented in the current sample, although slightly greater than those reported from the normative sample, are 
not consistent with levels of severe psychopathology that would be observed using psychiatric patient samples. The sample mean for the BSI GSI subscale score observed in this study fell between the $70^{\text {th }}$ and $84^{\text {th }}$ percentiles and was slightly above average, but likely not clinically noteworthy (Derogatis, 1993). Nevertheless, mental health symptoms that are within a normative range, like those observed in this study, are still associated with child distress observed during a 12 - 18 month immunization procedure.

Relation between adherence to immunization schedules and distress during 12 - 18 month

\section{immunizations}

Contrary to the hypotheses, the current study found no significant relations among adherence to child immunization schedules (as assessed via IDEA scores) and levels of child distress at a 12 - 18 month immunization procedure (as assessed by parent and nurse report or behavioral observation). With this sample, immunization schedule adherence was not associated with either behavioral observation of distress, parent report, or nurse report of child distress during a 12 - 18 month immunization procedure. These results failed to support either competing perspective that a) immunization schedule adherence provided the child with exposure to medical setting stimuli on a regular basis, thus resulting in progressive reduction in child distress behaviors at subsequent pediatric immunizations, or b) neutral stimuli associated with the immunization clinic setting could be conditioned to elicit conditioned emotional responses (i.e., child distress behaviors) with repeated pairings with aversive unconditioned stimuli (i.e., injection pain), thereby resulting in increased immunization distress in children from immunization-schedule-adherent families. There are several possible explanations for this unexpected lack of significant findings. 
Although some research suggests that instances of non-adherence to immunization schedules may be related to parental (Meyerhoff et al., 2001) and medical staff (Halperin, Eastwood, \& Halperin, 1998) concern about children's injection pain, no prior study has attempted to link adherence to levels of child procedural distress. It may be that parental and medical staff are concerned that injection pain impacts immunization schedule adherence; however, the data on this sample of 12 - 18 month old children suggest these concerns may not be warranted. That is, parental and medical staff concerns about injection pain may be informed by other variables (e.g., attitudes towards child pain or demographic variables) but may not be significantly associated with actual measures of child distress behavior during medical procedures. Thus, in the current sample, children who displayed more distress during a 12 - 18 month immunization procedure did not exhibit a history of non-adherence to immunization schedules.

Another explanation for the lack of findings linking adherence to measures of child distress pertains to the unique characteristics of the current sample with regard to adherence to immunization schedules. Average IDEA scores in the current study were approximately $30 \%$ lower (indicating lesser adherence) than values obtained in the initial validation study of this measure (Glauber, 2003). Thus, although almost all study participants were categorized as 'up-to-date' for purposes of immunization, it would seem that several of their previous immunization appointments occurred late. Additionally, because the current study examined children aged 12-18 months and the validation sample was comprised of records during 24month immunization visits, it is likely that IDEA scores may have been lower because they were calculated on only a few previous immunization appointments where multiple 
immunizations would have been given. One late appointment during these early immunization-heavy appointments could lead to inflated IDEA scores.

Finally, it is possible that adherence to immunization schedules may influence child distress, but not directly. As indicated by the observed significant interaction effects between adherence and both past medical distress and parent's attitudes regarding immunization, poorer adherence to immunization schedules did indeed relate to child distress, but only in children with histories of past medical distress or with parents with less adaptive attitudes regarding immunization.

Consistent with study hypotheses, children with negative prior experiences and low levels of immunization adherence were most at risk for displaying high levels of distress during a 12 - 18 month immunization procedure. However, it is noteworthy that this relation was not observed for predicting either parent or nurse report of child distress. It is possible that the interaction between prior medical distress and immunization schedule adherence is related to subtle differences in child distress behaviors that are difficult to detect via the parent and nurse report indices used in the present study but that are more readily apparent with an observational scale that may provide a more fine-grained analysis of distress behaviors.

There was also a significant interaction found among parent healthcare attitudes and adherence to immunization schedules in the prediction of observational child distress. Specifically, children of parents who reported maladaptive health care attitudes and low levels of immunization adherence displayed the highest levels of distress during a 12 - 18 month immunization procedure. Similar to the previous significant interaction, this finding was not observed in predicting either parent or nurse report of child distress. As mentioned 
previously, this lack of findings across parent and nurse reports of child distress may indicate that the interaction between health care attitudes and immunization schedule adherence affects more subtle child distress behaviors that are difficult to detect via the parent and nurse report forms used in the current investigation.

Contrary to hypotheses, the interactions between parent psychopathology and immunization schedule adherence in the prediction of child distress (via direct observation, parent, and nurse reports) were not significant. Although both prior medical distress and parent health care attitudes interacted with adherence to predict child distress, this was not the case for parent psychopathology and immunization schedule adherence. Also, because parent psychopathology was not correlated with measures of immunization schedule adherence, presence of psychological symptoms in parents did not impact their ability to schedule and obtain immunizations for their children. Whether such findings would be observed among parents diagnosed with psychiatric disorders remains a question for future research.

Measures of Child Distress during 12 - 18 Month Immunizations and Attendance at Future

\section{Immunization Appointment}

None of the three logistic regressions predicting adherence from distress were significant. This is not surprising, given that none of the univariate correlations between child distress measures and attendance at a future immunization appointment were significant. Thus, child distress during a 12 - 18 month immunization procedure did not influence attendance at a follow up immunization appointment. It is possible these unexpected findings are related to the rather simplistic dichotomous measure employed to assess follow-up attendance. Families' reasons for not attending the follow-up immunization 
appointment could have impacted the relation between the follow-up visit attendance and child distress measures. For example, it is probably important to distinguish families that did not attend the follow-up immunization appointment due to having a death in the family from those whose non-attendance was due to being "too busy" on the day of the appointment. However, because data regarding reasons for missing the follow-up visit proved extraordinarily difficult to obtain in the study sample, this type of analysis could not be conducted. Future studies will need to refine the strategy used in the current study for obtaining information pertaining to why the immunization visit was missed in order to more carefully examine these relations. Had study participants been asked why the scheduled immunization visit was cancelled when they eventually did visit the clinic on the next occasion, more complete data could have been collected. However, due to the multitude of dates and times for which future appointments were made and the number of appointments that were cancelled or missed and re-scheduled, it was impractical to have a member of the research team available to meet with each participating family on the exact day and time when they did return to the clinic.

\section{Limitations of study}

Although several factors linked with measures of child distress during immunizations were identified in this study, this study has several shortcomings that deserve discussion. First, the study sample was restricted with respect to geographical region in which data were collected as well as several other homogeneous characteristics of the sample (e.g., predominately Caucasian children from intact families of mostly low to middle class socioeconomic backgrounds). Thus the generalizability of these findings to other groups of children is questionable. 
Another limitation to this investigation is the relatively small sample size, which may have resulted in lower statistical power and ability to detect relations between variables in this sample when relations may have actually existed in the population. In other words, it is possible that some of the variables in the current investigation that lacked a significant relation to each other may have been significantly related if additional participants were included. In addition to the small sample size, a more diverse sample, particularly with regard to immunization schedule adherence, may have yielded greater variability in outcome measures, which also would have enhanced statistical power.

Missing data is another factor that may have influenced the outcomes in the current investigation. For example, due to invalid administrations of the measure of parent psychopathology (i.e., due to clear response bias displayed during measure completion), data for this measure were deleted for $14 \%$ of participants. This lack of data may have resulted in reduced power for the analyses involving parent psychopathology. Similarly, missing video data during the injections for a few children resulted in reduced power for all analyses using child behavior ratings.

A considerable number of univariate correlational analyses were conducted in the present investigation. It is possible that the number of statistical analyses conducted inflated the risk of type I error. Thus, some of the significant findings of the present investigation may have been due to chance (i.e., the null hypothesis may have been falsely rejected). Although the correlations observed in the present study reveal some important relations, future research is needed using larger samples in order to adopt more sophisticated multivariate statistical procedures. 
Another problem encountered during data collection involved the lack of consistency among medical records, making it difficult to measure other possible extraneous variables that may have assisted in interpreting information for determining immunization schedule adherence. A number of variables could influence adherence to schedules of immunization. For example, medical facilities sometimes reschedule immunization appointments due to scheduling conflicts with physician's schedules, child illness at the time of scheduled immunization, or a lack of immunization supplies. The retrospective chart review employed in this study to assess adherence history precluded an account of these or other possible confounding variables, as information regarding reasons for nonadherence were not included in the current participants' medical charts. In addition, although data regarding reasons for nonadherence were gathered with respect to families' attendance at a future immunization appointment, the limited amount of data that were actually gathered (e.g., due to a low sample size and considerable attrition) precluded these data from being included in statistical analyses. Future investigations of immunization schedule adherence should include an assessment of reasons for nonadherence, in order to account for these possible confounding variables.

Finally, the current investigation lacked an assessment of parent behaviors during their child's immunization procedure. The assessment of parent behaviors during pediatric immunization could have proved quite interesting given the important role parent psychopathology and parent health care attitudes seem to have in predicting child distress.

\section{Future directions and clinical implications}

Despite the limitations of this investigation, there are several tentative conclusions that can be drawn that have implications for treatment and prevention of child distress during 
pediatric immunizations. The data suggest that distress during a 12 - 18 month immunization procedure (per parent report) is related to the quality of children's prior medical experiences, with lower levels of distress associated with more positive quality previous medical experiences. In addition, immunization distress is related to child age such that younger children display higher levels of distress than older children. Thus, pain management protocols may be particularly helpful to implement during early infant immunizations in order to decrease rates of distress observed in younger children. Because negative prior medical experiences, particularly in conjunction with poor adherence to immunization schedules, may put children at risk for increased distress during a 12 - 18 month immunization, the use of pain management protocols during early immunizations may provide a buffer against distress at a $12-18$ month immunization by providing children with a more positive medical experience history.

The data also suggested that 12 - 18 month old boys exhibited (per direct observation) more distress than girls. The unexpected nature of these findings, coupled with the lack of findings across other measures of child distress (i.e., parent and nurse report), indicate that a more systematic investigation of the relation between gender and distress is warranted before implications are drawn for clinical practice.

A difficult child temperament was also associated with increased levels of child distress during a 12 - 18 month immunization (per parent report), suggesting that pain management interventions may be of particular benefit to young children with a more difficult temperament. However, distinct temperament dimensions were also associated with child distress, but none were consistent across all three measures of distress employed in the current investigation. Future studies should continue to examine the predictive value of a 
diagnostic cluster of temperament, as compared to that of individual temperament dimensions, to determine the most efficient way of identifying temperamental characteristics that may put children at risk for distress during immunization procedures. Pain management interventions may then be better targeted for delivery to these at-risk individuals.

Distress at a 12 - 18 month immunization (per parent report) was associated with parental health care attitudes so when maladaptive health care beliefs are endorsed by parents, child distress increases. Because maladaptive parental health care attitudes may put children at risk for increased immunization distress, these families may benefit from special counseling or support in the form of education regarding the function of pediatric health care. In addition, it is possible that pain management interventions that function to reduce child immunization distress may also have a positive impact on parental health care attitudes.

The data also show that levels of parent psychopathology were associated with child immunization distress; as immunization distress increased, so did levels of psychopathology. Moreover, this was the single most powerful predictor of child immunization distress, compared to all other predictors identified in the current investigation. Because this is the first study to explore this relation, future research is clearly needed to further examine variables that may impact the relation between parent psychopathology and child immunization distress. For example, specific parent behaviors during immunization procedures are likely associated with this indicator of psychopathology and should be elucidated. Subsequent instructional interventions aimed at guiding parent behaviors during immunization procedures or while preparing children for immunization procedures may provide a particularly effective path of intervention to reduce child immunization distress in families with parents exhibiting a greater frequency of psychological symptoms. 
Although these data suggest that immunization schedule adherence, via retrospective medical chart review, was not directly significantly associated with child immunization distress, adherence did influence observed child distress through its association with both prior medical experience and parent healthcare attitudes. That is, children with who have both negative prior medical experiences and low levels of immunization adherence display higher levels of observational distress during a 12 - 18 month immunization procedure. Similarly, children of parents who endorse maladaptive health care attitudes and have low levels of immunization adherence display higher levels of immunization distress. It is possible that interventions aimed at improving parent health care attitudes or reducing present or past child immunization distress may positively impact rates of immunization schedule adherence. Conversely, interventions aimed at improving rates of adherence may demonstrate indirect effects on child immunization distress. Clearly, due to the indirect effect that immunization schedule adherence has on child immunization distress, adherence to immunization schedules is an important variable that should be included in future examinations of child immunization distress, as these endeavors may document other possible interactive relations among adherence and other predictors of child immunization distress.

Continued investigation of predictors of pediatric immunization distress and adherence to pediatric schedules of immunization is essential. Immunization injections are the most common painful medical procedure of childhood (Ries, Roth, Syphan, Tarbell, \& Holubkov, 2003) and child distress associated with immunization procedures has clear immediate and lasting negative effects on the child, parent, and staff (e.g., Jacobson, Swan, Adegbenro, Ludington, Wollan, et al., 2001; PSRA, 1996). Multiple, effective 
pharmacological (e.g. local anesthetics, EMLA) and cognitive-behavioral interventions (for review see Piira et al., 2002) are currently available for pain management in pediatric settings; knowledge of predictors of immunization distress may extend the clinical application of these interventions to individuals who need them most. In addition, the discovery of variables predictive of immunization schedule adherence is important, given the benefits associated with timely pediatric immunizations, as well as the public health threat posed by immunization schedule nonadherence. 


\section{References}

Altshuler, J. L., \& Ruble, D. N. (1989). Developmental changes in children's awareness of strategies for coping with uncontrollable stress. Child Development, 60, 1337 1349.

American Academy of Pediatrics, Committee on Infectious Disease. (2003). Recommended childhood and adolescent immunization schedule. Pediatrics, 111, 212.

Anand, K. J., Coskun, V., Thrivikraman, K. V., Nemeroff, C. B., \& Plotsky, P. M. (1999). Long-term behavioral effects of repetitive pain in neonatal rat pups. Physiology and Behavior, 66, $627-637$.

Bachanas, P. J., \& Roberts, M. C. (1995). Factors affecting children's attitudes toward health care and responses to stressful medical procedures. Journal of Pediatric Psychology, 20, $261-275$.

Bakeman, R., \& Gottman, J. M. (1987). Applying observational methods: A systematic view. In J. D. Osofsky (Ed.). Handbook of Infant Development, Second edition (pp. 818 - 854). Oxford: Jon Wiley \& Sons.

Band, E. B. (1990). Children's coping with diabetes: Understanding the role of cognitive development. Journal of Pediatric Psychology, 15, 27 - 41.

Band, E. B., \& Wiesz, J. R. (1988). How to feel better when it feels bad: Children's perspectives on coping with everyday stress. Developmental Psychology, 24, 247 253.

Bates, J. E., Freeland, C. A., \& Lounsbury, M. L. (1979). Measurement of infant difficultness. Child Development, 50, $794-803$. 
Bijttebier, P., \& Vertommen, H. (1998). The impact of previous experience on children's reactions to venipunctures. Journal of Health Psychology, 3, 39- 46.

Blount, R. L., Piira, T., \& Cohen, L. L. (2003). Management of pediatric pain and distress due to medical procedures. In M. Roberts (Ed.). Handbook of pediatric psychology. $3^{\text {rd }}$ ed. (pp. 216 -233). New York: Guilford.

Bobo, J. K., Bale, J. L., Thapa, P. B., \& Wassilak, S. G. (1993). Risk factors for delayed immunization in a random sample of 1163 children from Oregon and Washington. Pediatrics, 91, $308-314$.

Carey, W. B. (1970). A simplified method for measuring infant temperament. Journal of Pediatrics, 77, $188-194$.

Centers for Disease Control and Prevention. (2003). Recommended childhood and adolescent immunization schedule - United States, 2003. Retrieved March 3, 2004, from http://www.cdc.gov/nip/recs/child-schedule.pdf

Cheng, S., Foster, R. L., \& Hester, N. O. (2003). A review of factors predicting children's pain experiences. Issues in Comprehensive Pediatric Nursing, 26, 203 - 216.

Cohen, J. (1960). A coefficient of agreement for nominal scales. Educational and Psychological Measurement, 20, 37 - 46.

Cohen, L. (2002). Reducing infant immunization distress through distraction. Health Psychology, 21, $207-211$.

Craig, K. D., McMahon, R. J., Morison, J. D., \& Zaskow, C. (1984). Developmental changes in infant pain expression during immunization injections. Social Science \& Medicine, 19, $1331-1337$. 
Dalqhuist, L. M., Gil, K. M., Armstrong, D., DeLawyer, D. D., Greene, P., \& Wuori, D. (1986). Preparing children for medical examinations: The importance of previous medical experience. Health Psychology, 5, 249 - 259.

Derogatis, L. R. (1993). Brief Symptom Inventory: Administration, Scoring, and Procedures Manual. $4^{\text {th }}$ ed. Minneapolis, MN: National Computer Systems, Inc.

Duncan, G. H., Bushnell, C. M., \& Lavigne, G. J. (1989). Comparison of verbal and visual analogue scales for measuring the intensity and unpleasantness of experimental pain. Pain, 37, $295-303$.

Finley, G. A., \& McGrath, P. J. (1998). The roles of measurement in pain management and research. In G. A. Finley \& P. J. McGrath (Eds.). Measurement of Pain in Infants and Children (pp. 1 - 4). Seattle: IASP Press.

Fullard, W., McDevitt, S. C., \& Carey, W. B. (1984). Assessing temperament in one- to three-year-old children. Journal of Pediatric Psychology, 9, 205 - 217.

Fyer, A. J., Mannuzza, S., Chapman, T., Martin, L. Y., \& Klein, D. F. (1995). Specificity in familial aggregation of phobic disorders, Archives of General Psychiatry, 52, 564573.

Goodenough, B., Kampel, L., Champion, G. D., Laubreaux, L., Nicholas, M. K., Ziegler, J. B., \& McInerney, M. (1997). An investigation of the placebo effect and age-related factors in the report of needle pain from venipuncture in children. Pain, 72, $383-$ 391.

Glauber, J. H. (2003). The immunization delivery effectiveness assessment score: A better immunization measure? Pediatrics, 112, $39-45$. 
Grunau, R. V. E., \& Craig, K. D. (1987). Pain expression in neonates: Facial action and cry. Pain, $28,395-410$.

Grunau, R. V. E., Whitefield, M. F., \& Petrie, J. H. (1994). Pain sensitivity and temperament in extremely low-birth-weight premature toddlers and preterm and fullterm controls. Pain, 58, $341-346$.

Guyer, B., Hughart, N., Holt, E., Ross, A., Stanton, B., et al. (1994). Immunization coverage and its relationship to preventative health care visits among inner-city children in Baltimore. Pediatrics, 94, $53-58$.

Hackworth, S. R., \& McMahon, R. J. (1991). Factor's mediating children's health care attitudes. Journal of Pediatric Psychology, 16, $69-85$.

Halperin, B. A., Eastwood, B. J., \& Halperin, S. A. (1998). Comparison of parental and health care professional preferences for the acellular or whole cell pertussis vaccine. Journal of Pediatric Infectious Diseases, 17, 103 - 109.

Harbeck, C. \& Peterson, L. (1992). Elephants dancing in my head: A developmental approach to children's concepts of specific pains. Child Development, 63, 138 149.

Hildegard, J., \& LeBaron, S. (1982). Relief of anxiety and pain in children and adolescents with cancer: Quantitative measures and clinical observations. International Journal of Clinical and Experimental Hypnosis, 30, 417 - 442.

Hubert, N. C., Jay, S. M., Saltoun, M., \& Hayes, M. (1988). Approach-avoidance and distress in children undergoing preparation for painful medical procedures. Journal of Clinical Child Psychology, 17, 194 - 202. 
Institute of Medicine. (2000). Calling the Shots - Immunization Finance Policies and Practices. Washington, DC: National Academy Press.

Izard, C. E., Hembree, E. A., Dougherty, L. M., \& Spizzirri, C. C. (1983). Changes in facial expressions of 2- to 19-month-old infants following acute pain. Developmental Psychology, 19, $418-426$.

Izard, C. E., Hembree, E. A., \& Huebner, R. R. (1987). Infants' emotion expressions to acute pain: Developmental change and stability of individual differences. Developmental Psychology, 23, 105 - 113.

Jacobsen, P. B., Manne, S. L., Gorfinkle, K., Schorr, O., Rapkin, B, \& Redd, W. H. (1990). Analysis of child and parent behavior during painful medical procedures. Health Psychology, 55, $860-865$.

Jacobson, R. M., Swan, A., Adegbenro, A., Ludington, S. L., Wollan, P. C., et al. (2001). Making vaccines more acceptable - Methods to prevent and minimize pain and other common adverse events associated with vaccines. Vaccine, 19, $2418-2427$.

Jay, S. M., Elliot, C. H., Katz, E. R., \& Siegel, S. E. (1987). Cognitive-behavioral and pharmacologic interventions for children's distress during painful medical procedures. Journal of Consulting and Clinical Psychology, 55, 860 - 865.

Jay, S. M., Ozolins, M., Elliot, C. H., \& Caldwell, S. (1983). Assessment of children's distress during painful medical procedures. Health Psychology, 2, 133 - 147.

Katz, E. R., Kellerman, J., \& Siegel, S. E. (1980). Behavioral distress in children with cancer undergoing medical procedures: Developmental considerations. Journal of Consulting and Clinical Psychology, 48, 356-365. 
LeBaron, S., \& Zelter, L. (1984). Assessment of acute pain and anxiety in children and adolescents by self-reports, observer reports, and a behavior checklist. Journal of Consulting and Clinical Psychology, 52, $729-738$.

Lee, L. W., \& White-Traut, R. C. (1996). The role of temperament in pediatric pain response. Issues in Comprehensive Pediatric Nursing, 19, 49 - 63.

Lumley, M. A., Melamed, B. G., \& Abeles, L. A. (1993). Predicting children's presurgical anxiety and subsequent behavior changes. Journal of Pediatric Psychology, $18,481-497$.

MacLaren, J. E., \& Cohen, L. L. (2004, May). The procedural pain attitudes scale: Relations among parent attitudes, parent anxiety, and child pain. Poster session presented at the Annual meeting of the Canadian Pain Society, Vancouver, Canada.

MacLaren, J. E., McCourt, G., \& Cohen, L. L. (2004, April). Parent health care attitudes and children's procedural distress. Poster session presented at the Society for Pediatric Psychology National Conference on Child Health Psychology, Charleston, SC.

Maier, W., Lichtermann, D., Kazdin, A., Orvaschel, H., \& Perrin, S. (1993). A controlled family study in panic disorder. Journal of Psychiatry Research, 27, $79-87$.

McAllister, W. R., \& McAllister, D. E. (1995). Two-factor fear theory: Implications for understanding anxiety-based clinical phenomena. In W. T. O'Donohue \& L. Krasner, (Eds.). Theories of behavior therapy: Exploring behavior change (pp. 145-171). Washington, DC: American Psychological Association. McGrath, P. J. (1990). Pain in children: Nature, assessment, and treatment. New York: Guilford Press. 
McGrath, P. J., Johnson, G. Goodman, J. T., Schillinger, J., Dunn, J., \& Chapman, J. A. (1985). CHEOPS: A behavioral scale for rating postoperative pain in children. In $\mathrm{H}$. L. Field, R. Dubner, \& F. Cervero (Eds.). Advances in Pain Research and Therapy: Volume Nine, Proceedings of the Fourth World Congress on Pain (pp. 395 - 402). New York: Raven Press.

Megel, M. E., Houser, C. W., \& Gleaves, L. S. (1998). Children’s responses to immunizations: Lullabies as a distraction. Issues in Comprehensive Pediatric Nursing, 21, $129-145$.

Melamed, B. G., \& Siegel, L. J. (1985). Children's reactions to medical stressors: An ecological approach to the study of anxiety. In A. H. Tuma \& J. D. Maser (Eds.) Anxiety and its disorders (pp. 369 - 386). Hillsdale, NJ: Erlbaum.

Melzack, R. (1999). From the gate to the neuromatrix. Pain Supplement, 6, S121 - S126. Mendlewicz, J., Papadimitriou, G., \& Wilmotte, J. (1993). Family study of panic disorder: Comparison with generalized anxiety disorder, major depression, and normal subjects. Psychiatry and Genetics, 3, $73-78$.

Merskey, H., \& Bogduk, N. (Eds). (1994). Classification of chronic pain: Descriptions of chronic pain syndromes and definitions of pain terms: Second edition (pp. 209-214). Seattle: IASP Press.

Meyerhoff, A. S., Weniger, B. G., \& Jacobs, J. (2001). Economic value to parents of Reducing the pain and emotional distress of childhood vaccine injections. Pediatric Infectious Disease Journal, 20, 57 - 62. 
McDevitt, S. C., \& Carey, W. B. (1978). The measurement of temperament in three to seven year old children. Journal of Child Psychology \& Psychiatry, 19, 245 253.

Mowrer, O. H. (1947). On the dual nature of learning - a reinterpretation of “conditioning” and "problem-solving." Harvard Educational Review, 17, 102 - 148.

Pate, J. T., Blount, R. I., Cohen, L. L., \& Smith, A. J. (1996). Childhood medical experience and temperament as predictors of adult functioning in medical situations. Children's Health Care, 25, 281 - 296.

Piira, T., Hayes, B., \& Goodenough, B. (2002). Distraction methods in the management of children's pain: An approach based on evidence or intuition? The Suffering Child, $1,1-10$.

Princeton Survey Research Associates. (1996). Benchmark surveys on childhood immunization. Princeton, NJ: Gallup Poll.

Prislin, R., Dyer, J. A., Blakely, C. H., \& Johnson, C. D. (1998). Immunization status and sociodemographic characteristics: The mediating role of beliefs, attitudes, and perceived control. American Journal of Public Health, 88, 1821 - 1826.

Reis, E. C. (1997). Multiple scheduled injections contribute to missed opportunities to immunize during well care visits. Ambulatory Child Health, 3, 172.

Reis, E. C., Roth, E. K., Syphan, J. L., Tarbell, S. E., \& Holubkov, R. (2003). Effective pain reduction for multiple immunization injections in young infants. Archives of Pediatrics and Adolescent Medicine, 157, 1115 - 1120. 
Rudolph, K. D., Dennig, M. D., \& Weisz, J. R. (1995). Determinants and consequences of children's coping in the medical setting: Conceptualization, review, and critique. Psychological Bulletin, 118, $328-357$.

Schechter, N. L., Berstein, B. A., Beck, A., Hart, L., \& Scherzer, L. (1991). Individual differences in children's response to pain: Role of temperament and parental characteristics. Pediatrics, 87, $171-177$.

Stein, M., Chartier, M. J., Hazen, M. L., Kozak, M. V., Tancer, M. E., Lander, S., Furer, P., Chubaty, D. \& Walker, J. R. (1998). A direct-interview family study of generalized social phobia. American Journal of Psychiatry, 155, 90-97.

Sufka, K. J., \& Price, D. D. (2002). Gate control theory reconsidered. Brain and Mind, 3, 277- 280.

Sweet, S. D., \& McGrath, P. J. (1998). Relative importance of mother's versus medical staff's behavior in the prediction of infant immunization pain behavior. Journal of Pediatric Psychology, 23, $249-256$.

Sweet, S. D., McGrath, P. J., \& Symons, D. (1999). The roles of child reactivity and parenting context in infant pain response. Pain, 80, $655-661$.

Szilagyi, P. G., Rodewald, L. E., \& Savageau, J. (1992). Improving vaccination rates in children with asthma: A test of a computerized reminder system and an analysis of factors predicting vaccination compliance. Pediatrics, $6,871-875$.

Taddio, A., Goldbach, M., Ipp, M., Stevens, B., \& Koren, G. (1995). Effect of neonatal circumcision on pain response during vaccination in boys. Lancet, 345, $291-292$.

Taddio, A., Nulman, I., Goldbach, M., Ipp, M., \& Koren, G. (1994). Use of lidocaine cream for vaccination pain in infants. The Journal of Pediatrics, 124, $643-648$. 
Taddio, A., Nulman, I., Koren, B. S., Stevens, B., \& Koren, G. (1995). A revised measure of acute pain in infants. Journal of Pain and Symptom Management, 10, $456-$ 463.

Thomas, A., \& Chess, S. (1977). Temperament and development. Oxford: Brunner/Mazel.

Varni, J. W., Walco, G. A., \& Wilcox, K. T. (1990). Cognitive-biobehavioral assessment and treatment of pediatric pain. In A. M. Gross \& R. S. Drabman (Eds.). Handbook of Clinical Behavioral Pediatrics. New York: Plenum

Weisz, J. R., McCabe, M., \& Dennig, M. D. (1994). Primary and secondary control among children undergoing medical procedures: Adjustment as a function of coping style. Journal of Consulting and Clinical Psychology, 62, 324 - 332.

Wood, D. Donald-Sherbourne, C., Halfon, N., Tucker, M. B., Oritz, V., et al. (1995). Factors related to immunization status among inner-city Latino and African American preschoolers. Pediatrics, 96, 295 - 301. 
Appendix A

\section{PATIENT INFORMATION FORM}

Date Participant \#

\section{CHILD INFORMATION:}

Child AGE:

DATE OF BIRTH:

SEX (circle): Male Female

GRADE:

Your relationship to the Child (Patient): ) RACE (circle): Caucasian African-American Asian-American Hispanic-American Other:

Your AGE years

Child's Hometown: County:

Average Travel Time to this medical facility: Minutes

Who USUALLY cares takes your child to receive immunization injections? Mother Father Other (Relation to child?

Does your child have any illnesses?

No Yes (Please List: 
Who regularly takes care of your child's medical care? For example, who routinely takes him/her to medical appointments, fills prescriptions, and so on?

Mother

Father

Other (Relation to child?

\section{FAMILY INFORMATION:}

Please make a check in front of each family member that is CURRENTLY living in your child's home.

$\begin{aligned} & \text { Biological Mother } \\ & \text { Adoptive or Step-Mother } \\ & \text { Brother (How many? } \\ & \begin{array}{l}\text { Grandparent (How many? } \\ \text { Other (list by relationship to child) }\end{array}\end{aligned}-\begin{aligned} & \text { Biological Father } \\ & \text { Adoptive or Step-Father } \\ & \text { Sister (How many? }\end{aligned}$

Please make a check in front of your marital status.

Never Been Married / Single

Married to other biological parent

Living with boyfriend / girlfriend
Divorced / Single

Remarried to step-parent

Widowed (other biological parent is deceased)

Please check your total, annual family income level:

Less than $\$ 15,000$

$\$ 15,000$ - $\$ 24,999$

$\$ 25,000-\$ 34,999$

$\$ 35,000$ - $\$ 50,000$

Greater than $\$ 50,000$

Please check your child's (the patient) INSURANCE:

None/My child is NOT insured

Medicaid

Other (Provide Company Name: 
The highest education level you attained (Check one only):

Middle School College Freshman

College Senior
Some High School College Sophomore Bachelor's Degree
High School Graduate College Junior Master's Degree __ Doctorate

The highest education level your spouse attained (Check one only): Middle School_Some High School_High School Graduate College Freshman College Sophomore College Junior

__ College Senior__ Bachelor's Degree __ Master's Degree __ Doctorate

Please provide a job title \& description for you and your spouse.

Mother (or other, please specify):

Father (or other, please specify): 
Appendix B

\section{Past Medical Experiences Questionnaire}

Participant \# Date

\section{For the following questions, please think back to the first time your child received an immunization injection.}

How distressed were you during your child's first injection?

Not Distressed

Very Distressed

How distressed was your child during his/her first injection?

Not Distressed

Very Distressed

For the following questions, please think about all the times your child has experienced the following medical procedures.

How distressed was your child during past throat cultures?

Not Distressed

Very Distressed

How distressed was your child during past medical appointments?

Not Distressed

Very Distressed

How distressed was your child during past dental appointments?

Not Distressed

Very Distressed

How distressed was your child during past hospitalizations?

Not Distressed

Very Distressed 


\section{Appendix C}

(see attached VAS Questionnaires) 


\title{
Clinician Post-Injection Questionnaire
}

\author{
Participant \# \\ Date
}

How distressed were you during this child's injection?

Not Distressed

Very Distressed

How distressed was this parent during this child's injection?

Not Distressed

Very Distressed

How distressed was this child during the injection?

Not Distressed

Very Distressed 


\title{
Caregiver Post-Injection Questionnaire
}

\author{
Participant \#___ Date
}

How distressed were you during your child's injection?

Not Distressed

Very Distressed

How distressed was your child during the injection?

Not Distressed

Very Distressed 
Appendix D

Reasons for Nonattendance Form

Child's Name:

Parent's Name:

Please complete the following to provide a better understanding of the difficulties families experience in attending their children's vaccination appointments.

\section{Missed immunization appointment because:}

Scheduling conflict

(for example: too busy, had another appointment)

Family member sick

(for example: child, sibling, or parent was sick)

Family emergency

Forgot

Other

(please explain: 


\section{Table 1}

$\underline{\text { Frequencies (and Percentages) for Demographic Variables }}$

\begin{tabular}{ll}
\hline Variable & $\mathrm{n}(\%)$ \\
\hline Child Characteristics & 50 \\
Gender & $24(48)$ \\
Boy & $26(52)$ \\
Girl & \\
Race & \\
Caucasian & $39(78)$ \\
African-American & $4(8)$ \\
Asian American & $2(4)$ \\
Hispanic American & 1 \\
Other & $46(9)$ \\
Foather & $3(6)$
\end{tabular}


Variable $\mathrm{n}(\%)$

Child Medical and Immunization History

Number of injections received at study

Enrollment

One

Two

$30(60)$

Three

$12(24)$

Five

$4(8)$

Presence of chronic illness

Yes

4

No

46

Child insurance

Yes

50

No

0

Family Characteristics

Parents' marital status ${ }^{\mathrm{b}}$

Married to biological parent of child

or step-parent

Single parent, divorced / never been

Married

Living together, unmarried

$7(14)$

(table continues) 
Variable

Total Annual Family Income ${ }^{c}$

Less than $\$ 15,000$

$\$ 15,000-\$ 34,999$

$\$ 35,000-\$ 50,000$

Greater than $\$ 50,000$

Education of Father $^{\mathrm{d}}$

High school graduate or less

Some college

Bachelor's degree

Post graduate degree

Education of Mother $^{\text {a }}$

High school graduate or less

Some college

Bachelor's degree

Post graduate degree

Occupation of father e

White collar workers (i.e., professional, managerial, or administrative positions)

Unemployed/Disabled/Student

Unskilled / Skilled Laborer n $(\%)$

$19(38)$

$14(28)$

$8(16)$

$6(12)$

$22(44)$

$6(12)$

$6(12)$

9 (18)

$23(46)$

$13(26)$

7 (14)

$6(12)$

13 (26)

$22(24)$

$6(12)$

(table continues) 
Variable

Occupation of mother $^{\mathrm{d}}$

White collar workers (i.e., professional, managerial, or administrative positions)

Unemployed/Disabled/Student

Unskilled / Skilled Laborer

$19(38)$

${ }^{\mathrm{a}}$ Missing data, $\mathrm{n}=1$

${ }^{\mathrm{b}}$ Missing data, $\mathrm{n}=2$

${ }^{\mathrm{c}}$ Missing data, $\mathrm{n}=3$

${ }^{\mathrm{d}}$ Missing data, $\mathrm{n}=7$

${ }^{\mathrm{e}}$ Missing data, $\mathrm{n}=9$ 
Table 2

Means and Standard Deviations for Demographic Variables

Variable

$\mathrm{M}(\mathrm{SD})$

Child

Age (months)

$14(2.58)$

Parent

Age (years)

Child Medical and Immunization History

Child distress at first injection ${ }^{\mathrm{a}} \quad 5.38$ (3.58)

Parent distress at child's first injection ${ }^{\text {a }} \quad 5.46$ (3.57)

Child overall past medical distress $^{\mathrm{a}} \quad 4.06(2.25)$

Number of medical visits in past 6 months $\quad .86(.68)$

Number of months since last medical visit $\quad 5.27$ (1.94)

Travel time to medical clinic (miles) 23.51 (16.71)

Family Characteristics

Number of persons living in home 4.13 (1.40)

${ }^{a}$ Higher scores indicate greater distress; maximum possible score is 10 
Table 3

Descriptive Statistics for Measures of Distress

$\underline{\mathrm{n}}$

$\underline{\mathrm{M}}$

$\underline{\mathrm{SD}}$

Modified Behavioral Pain Scale (MBPS) ${ }^{\text {a }}$

Baseline score

36

1.34

.61

Pre-injection score

43

1.50

.70

Injection score

48

2.38

.49

Recovery score

41

1.57

.48

Visual Analog Scale (VAS) Ratings of Distress ${ }^{b}$

Parent report of child distress

50

6.8

Nurse report of child distress

50

6.8

Parent self-rated distress

50

4.22

Nurse report of parent distress

50

2.7

2.45

Nurse self-rated distress

50

.45

.79

${ }^{a}$ Higher scores indicate greater distress; maximum possible score is 3.33

${ }^{\mathrm{b}}$ Higher scores designate more distress; maximum possible score is 10 
Table 4

Descriptive Statistics for Measures of Child Temperament

$\underline{\mathrm{n}} \quad \underline{\mathrm{M}} \quad \underline{\mathrm{SD}}$

TTS activity subscale score

50

3.74

.50

TTS rhythmicity subscale score

50

2.92

.59

TTS approach subscale score

50

3.23

.83

TTS adaptability subscale score

50

3.26

.64

TTS intensity subscale score

50

3.99

.49

TTS mood subscale score

50

3.12

.59

TTS persistency subscale score

50

3.47

.44

TTS distractibility subscale score

50

4.01

.53

TTS threshold subscale score

50

3.84

.70

Diagnostic cluster

50

Easy

22

Slow-to-warm-up

13

Difficult

15

Note. Higher scores indicate more difficult temperament characteristics. 
Table 5

Descriptive Statistics for Measures of Health Care Beliefs

\begin{tabular}{lccc}
\hline & $\underline{\mathrm{n}}$ & $\underline{\mathrm{M}}$ & $\underline{\mathrm{SD}}$ \\
\hline Total HCIAQ score $^{\mathrm{a}}$ & 49 & 45.41 & 6.27 \\
Procedural anxiety and pain subscale score & 49 & 23.33 & 4.60
\end{tabular}

${ }^{a}$ Higher scores indicate more adaptive parental immunization beliefs; maximum possible score is 60 . 
Table 6

Descriptive Statistics for Measures of Parent Psychopathology

\begin{tabular}{lccc}
\hline & $\underline{n}$ & $\underline{M}$ & $\underline{\text { SD }}$ \\
& & & .36 \\
BSI somatization raw score & 43 & .42 & .78 \\
BSI obsessive-compulsive raw score & 43 & .95 & .59 \\
BSI interpersonal sensitivity raw score & 43 & .59 & .42 \\
BSI depression raw score & 43 & .34 & .38 \\
BSI anxiety raw score & 43 & .38 & .45 \\
BSI hostility raw score & 43 & .49 & .30 \\
BSI phobic anxiety raw score & 43 & .18 & .66 \\
BSI paranoid ideation raw score & 43 & .60 & .44 \\
BSI psychoticism raw score & 43 & .31 & .44 \\
BSI global severity index raw score & 43 & .51 &
\end{tabular}

Note. Higher scores indicate more psychopathology; maximum score is 3 
Table 7

Descriptive Statistics for Measures of Adherence to Immunization Schedule

$\underline{\mathrm{n}} \quad \underline{\mathrm{M}}$

$\underline{\mathrm{M}} \quad \underline{\mathrm{SD}}$

Up-to-date immunization status

48

Up-to-date

47

Not up-to-date

1

IDEA composite score $^{\mathrm{a}} \quad 48$

50

Arrival at follow up immunization visit

50

Yes

25

No

25

${ }^{\text {a }}$ Higher scores indicate greater adherence; maximum possible score is 1 
Table 8

Correlations between Demographic Variables and Child Distress Variables

\begin{tabular}{llll}
\hline Demographic Variable & $\begin{array}{l}\text { Observational } \\
\text { distress }\end{array}$ & $\begin{array}{l}\text { Parent rating } \\
\text { child distress }\end{array}$ & $\begin{array}{l}\text { Nurse rating } \\
\text { child distress }\end{array}$ \\
\hline Child Characteristics & & $-.32^{*}$ & -.05 \\
Age (months) & $-.30^{*}$ & -.08 & -.16 \\
Gender & .24 & $.33^{*}$ & $.38^{* *}$ \\
Race & & & \\
Parent Characteristic & & -.09 & -.21
\end{tabular}

Child Medical and Immunization History

Child distress at first injection $\quad .08$

$.08 \quad .24$

$\begin{array}{llll}\text { Parent distress at child's first injection } & -.07 & .24 & .21\end{array}$

$\begin{array}{llll}\text { Child overall past medical distress } & .05 & .30 * & .25\end{array}$

$\begin{array}{llll}\text { Number of medical visits in past } 6 \text { months } & -.06 & .04 & .05\end{array}$

$\begin{array}{llll}\text { Time since last medical visit } & -.16 & .02 & -.10\end{array}$

$\begin{array}{llll}\text { Number of injections received during clinic } & -.05 & .20 & .07\end{array}$

Visit

$\begin{array}{llll}\text { Travel time to medical clinic } & -.03 & -.09 & .01\end{array}$

Family Characteristics

$\begin{array}{llll}\text { Number of persons living at home } & .11 & .17 & .20 \\ \text { Parents' marital status } & .13 & -.08 & -.07\end{array}$

(table continues) 
Table 8 (continued)

\begin{tabular}{llll}
\hline Demographic Variable & $\begin{array}{l}\text { Observational } \\
\text { distress }\end{array}$ & $\begin{array}{l}\text { Parent rating } \\
\text { child distress }\end{array}$ & $\begin{array}{l}\text { Nurse rating } \\
\text { child distress }\end{array}$ \\
\hline Total Annual Family Income & -.10 & -.24 & -.17 \\
Education of Father & -.07 & -.20 & .01 \\
Education of Mother & -.09 & -.03 & -.20 \\
Occupation of father & .29 & .21 & -.05 \\
Occupation of mother & .23 & -.04 & -.07 \\
$* p<.05$ & & & \\
$* * p<.01$ & & &
\end{tabular}


Table 9

Correlations between Child Temperament Scales and Child Distress Variables

Variable

$\begin{array}{lll}\text { Observ } & \text { Parent } & \text { Nurse } \\ \text { ational } & \text { rating } & \text { rating } \\ \text { distress } & \text { child } & \text { child } \\ & \text { distress } & \text { distress }\end{array}$

TTS activity subscale score

$-.02$

$-.06$

.14

TTS rhythmicity subscale score

$.20 \quad .30 *$

.27

TTS approach subscale score

.06

$.32 *$

.16

TTS adaptability subscale score

$.32 *$

$.38 *$

.18

TTS intensity subscale score

$-.01$

.01

.05

TTS mood subscale score

$.35 *$

.25

$* .30$

TTS persistency subscale score

.02

.05

.22

TTS distractibility subscale score

$-.01$

$-.14$

.04

TTS threshold subscale score

$.31 *$

.11

.18

Diagnostic cluster

$-.17$

$-.46^{* *}$

$-.23$

$* p<.05$

$* * p<.01$ 
Table 10

Correlations between Measures of Health Care Beliefs and Child Distress Variables

\begin{tabular}{lcccc}
\hline Variable & $\begin{array}{l}\text { Observ } \\
\text { ational } \\
\text { distress }\end{array}$ & $\begin{array}{l}\text { Parent } \\
\text { rating } \\
\text { child } \\
\text { distress }\end{array}$ & $\begin{array}{l}\text { Nurse } \\
\text { rating } \\
\text { child } \\
\text { distress }\end{array}$ \\
\hline Total HCIAQ score & -.11 & $-.42^{* *}$ & -.09 \\
Procedural anxiety and pain subscale score & -.17 & $-.42^{* *}$ & -.14 \\
\hline
\end{tabular}

$* * p<.01$ 
Table 11

Correlations between Measures of Parent Psychopathology and Child Distress Variables

\begin{tabular}{|c|c|c|c|}
\hline Variable & $\begin{array}{l}\text { Observ } \\
\text { ational } \\
\text { distress }\end{array}$ & $\begin{array}{l}\text { Parent } \\
\text { rating } \\
\text { child } \\
\text { distress }\end{array}$ & $\begin{array}{l}\text { Nurse } \\
\text { rating } \\
\text { child } \\
\text { distress }\end{array}$ \\
\hline BSI somatization score & $.32 *$ & .27 & .26 \\
\hline BSI obsessive-compulsive score & .15 & .25 & .09 \\
\hline BSI interpersonal sensitivity score & $.37 *$ & $.31 *$ & .26 \\
\hline BSI depression score & .21 & .27 & .22 \\
\hline BSI anxiety score & $.33 *$ & $.38^{*}$ & $.42 * *$ \\
\hline BSI hostility score & .30 & .24 & .15 \\
\hline BSI phobic anxiety score & $.32 *$ & .19 & .22 \\
\hline BSI paranoid ideation score & $.39 *$ & .26 & .24 \\
\hline BSI psychoticism score & $.50 * *$ & .28 & $.31 *$ \\
\hline BSI global severity index score & $.41 * *$ & $.31 *$ & .30 \\
\hline
\end{tabular}

Note. $n=43$

$* p<.05$

$* * p<.01$ 
Table 12

Correlations between Measure of Adherence to Immunization Schedule and Child Distress Variables

\begin{tabular}{lllll}
\hline Variable & $\begin{array}{l}\text { Observ Parent } \\
\text { ational } \\
\text { distress } \begin{array}{l}\text { rating } \\
\text { child } \\
\text { distress }\end{array}\end{array}$ & $\begin{array}{l}\text { Nating } \\
\text { child } \\
\text { distress }\end{array}$ \\
\hline IDEA composite score & & & & \\
\hline
\end{tabular}


Table 13

Multiple Regression Analysis with MBPS Score: Child Age, Child Gender, Child

Temperament, Parent Health Beliefs, Prior Medical Experience, and Parent Psychopathology

Variable

F $\quad R^{2}$

$\beta$

$t$

$p$

Child Age

Child Gender

TTS Diagnostic Cluster

HCIAQ Subscale

Prior Distress

BSI Global Severity Index

$\begin{array}{llll}\text { Full Model } & 2.59 & .02 & .34\end{array}$

$-.33$

$-.25$

.07

$-.11$

$\begin{array}{ll}-.04 & -.27\end{array}$

.79

$\begin{array}{lll}.39 & 2.54 \quad .02\end{array}$

.03

.09

.64

.50

79 
Table 14

Multiple Regression Analysis with Parent Rating of Child Distress: Child Age, Child Gender, Child Temperament, Parent Health Beliefs, Prior Medical Experience, and Parent

Psychopathology

Variable

F $\quad p$

$R^{2}$

$\beta$

$t$

$p$

Child Age

Child Gender

TTS Diagnostic Cluster

HCIAQ Subscale

Prior Distress

BSI Global Severity Index

Full Model
.06

$-.06$

$-.38$

$-.28$

.15

.09
.44

$-.45$

$-2.55$

.02

$-1.87$

1.03

.60

.55

.66

.66
.37 
Table 15

Multiple Regression Analysis with Nurse Rating of Child Distress: Child Age, Child Gender, Child Temperament, Parent Health Beliefs, Prior Medical Experience, and Parent

Psychopathology

Variable

$F$

$p$

$R^{2}$

$\beta$

$t$

$p$

Child Age

Child Gender

TTS Diagnostic Cluster

HCIAQ Subscale

Prior Distress

BSI Global Severity Index

Full Model
1.4
$-.17$

$-1.06$

$\begin{array}{ll}-.16 & -1.05\end{array}$

$-.11$

$-.63$

.12

1.14

1.37
.02

.19

.23

.19

.30

.30

.53

.91

.26

.18 
Table 16

Hierarchical Multiple Regression Analysis with MBPS Injection Score: Interaction of Prior $\underline{\text { Medical Experience and Adherence to Immunization Schedule }}$

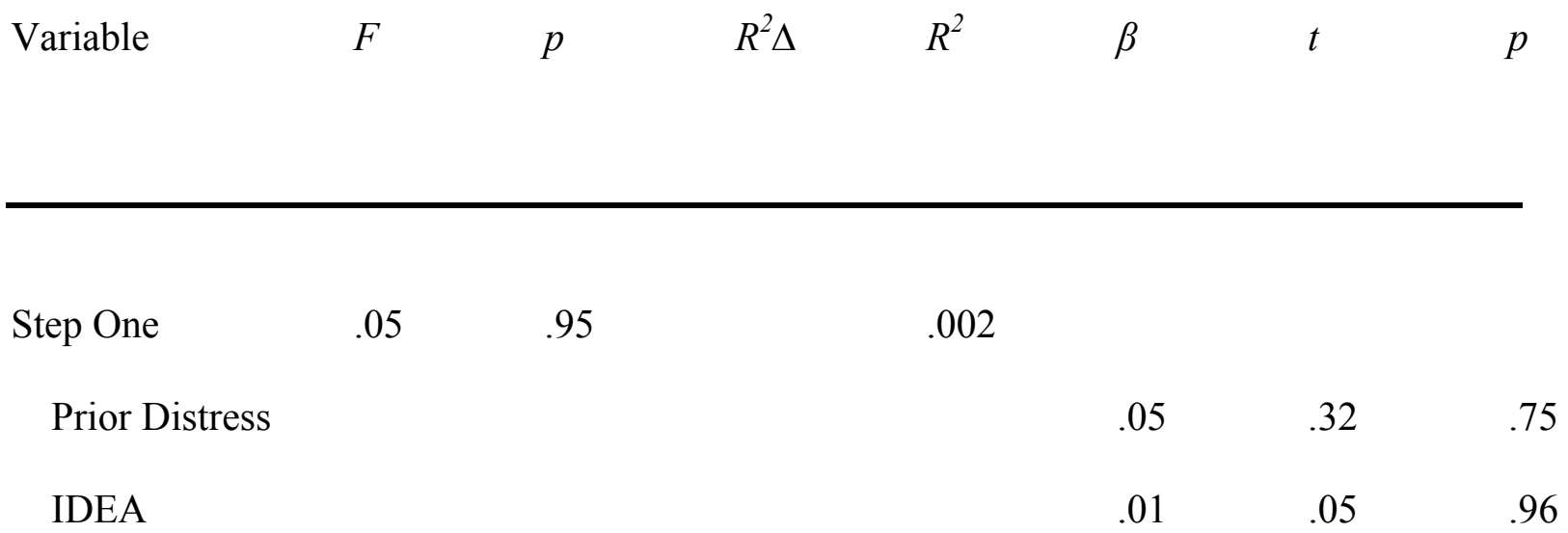

Step Two

Prior Distress x $\quad 6.71 \quad .03 \quad .14$

IDEA 
Table 17

Hierarchical Multiple Regression Analysis with Parent Rating of Child Distress: Interaction of Prior Medical Experience and Adherence to Immunization Schedule

$\begin{array}{llllllll}\text { Variable } & F & p & R^{2} \Delta & R^{2} & \beta & t\end{array}$

$\begin{array}{llll}\text { Step One } & 2.31 & .11 \\ \text { Prior Distress } & & & \\ \text { IDEA } & & & \\ \text { Step Two } & & & .00 \\ \text { Prior Distress x } & .006 & .90 & \\ \text { IDEA } & & & \end{array}$

$\begin{array}{lll}.29 & 2.01 & .05 \\ -.08 & -.56 & .58\end{array}$

.10 
Table 18

Hierarchical Multiple Regression Analysis with Nurse Rating of Child Distress: Interaction of Prior Medical Experience and Adherence to Immunization Schedule

\begin{tabular}{llllllll} 
Variable & $F$ & $p$ & $R^{2} \Delta$ & $R^{2}$ & $\beta$ & $t$ & $p$ \\
\hline
\end{tabular}

$\begin{array}{llllll}\text { Step One } & 1.55 & .22 & .06 & & \\ \\ \text { Prior Distress } & & .25 & 1.7 & .10 \\ \text { IDEA } & & & -.04 & -.26 & .80\end{array}$

Step Two

Prior distress $\mathrm{X} \quad 1.02 \quad .83 \quad .02$

IDEA 
Table 19

Hierarchical Multiple Regression Analysis with MBPS Injection Score: Interaction of Parent $\underline{\text { Health Beliefs and Adherence to Immunization Schedule }}$

$\begin{array}{llllllll}\text { Variable } & F & p & R^{2} \Delta & R^{2} & \beta & t & \\ & & & \\ \end{array}$

$\begin{array}{llllll}\text { Step One } & .05 & .53 & .03 & & \\ \text { HCIAQ } & & & -.17 & -1.13 & .27 \\ \text { IDEA } & & & -.003 & -.02 & .98 \\ \text { Step Two } & & .02 & .11 & & \\ \text { HCIAQ x IDEA } & 5.37 & .02 & & \\ \end{array}$


Table 20

Hierarchical Multiple Regression Analysis with Parent Rating of Child Distress: Interaction of Parent Health Beliefs and Adherence to Immunization Schedule

$\begin{array}{llllllll}\text { Variable } & F & p & R^{2} \Delta & R^{2} & \beta & t\end{array}$

$\begin{array}{llllll}\text { Step One } & 5.09 & .01 & .19 & & \\ \\ \text { HCIAQ } & & -.42 & -3.1 & .004 \\ \text { IDEA } & & -.12 & -.91 & .37\end{array}$

Step Two

HCIAQ x IDEA $\quad 3.7 \quad .12 \quad .02$


Table 21

Hierarchical Multiple Regression Analysis with Nurse Rating of Child Distress: Interaction of Parent Health Beliefs and Adherence to Immunization Schedule

$\begin{array}{llllllll}\text { Variable } & F & p & R^{2} \Delta & R^{2} & \beta & t\end{array}$

$\begin{array}{llllll}\text { Step One } & .52 & .60 & .02 & & \\ \\ \text { HCIAQ } & & -.14 & -.93 & .36 \\ \text { IDEA } & & -.07 & -.45 & .65\end{array}$

Step Two

HCIAQ x IDEA $1.92 \quad .38 \quad .04$ 
Table 22

Hierarchical Multiple Regression Analysis with MBPS Injection Score: Interaction of Parent $\underline{\text { Psychopathology and Adherence to Immunization Schedule }}$

\begin{tabular}{|c|c|c|c|c|c|c|c|}
\hline Variable & $F$ & $p$ & $R^{2} \Delta$ & $R^{2}$ & $\beta$ & $t$ & $p$ \\
\hline Step One & 4.09 & .03 & & .19 & & & \\
\hline BSI global & & & & & .46 & 2.86 & .007 \\
\hline \multicolumn{8}{|c|}{ severity index } \\
\hline IDEA & & & & & -.15 & -.94 & .36 \\
\hline \multicolumn{8}{|l|}{ Step Two } \\
\hline BSI-GSI x & 1.05 & .07 & .02 & & & & \\
\hline
\end{tabular}


Table 23

Hierarchical Multiple Regression Analysis with Parent Rating of Child Distress: Interaction of Parent Psychopathology and Adherence to Immunization Schedule

$\begin{array}{llllllll}\text { Variable } & F & p & R^{2} \Delta & R^{2} & \beta & t & \\ & & & \\ \end{array}$

\begin{tabular}{|c|c|c|c|c|c|c|}
\hline Step One & 3.22 & .05 & & & & \\
\hline BSI global & & & & .39 & 2.43 & .02 \\
\hline \multicolumn{7}{|c|}{ severity index } \\
\hline IDEA & & & & -.24 & -1.50 & .14 \\
\hline \multicolumn{7}{|l|}{ Step Two } \\
\hline BSI-GSI x & .02 & .73 & .001 & & & \\
\hline
\end{tabular}


Table 24

Hierarchical Multiple Regression Analysis with Nurse Rating of Child Distress: Interaction of Parent Psychopathology and Adherence to Immunization Schedule

$\begin{array}{llllllll}\text { Variable } & F & p & R^{2} \Delta & R^{2} & \beta & t & \\ & & & \\ \end{array}$

\begin{tabular}{|c|c|c|c|c|c|c|}
\hline Step One & 2.5 & .10 & & & & \\
\hline BSI global & & & & .36 & 2.2 & .03 \\
\hline \multicolumn{7}{|c|}{ severity index } \\
\hline IDEA & & & & -.18 & -1.12 & .27 \\
\hline \multicolumn{7}{|l|}{ Step Two } \\
\hline BSI-GSI x & .40 & .23 & .01 & & & \\
\hline
\end{tabular}


Table 25

$\underline{\text { Correlations between Attendance at Follow-Up Visit and Demographic Variables }}$

Demographic Variable

Attendance at follow-up visit ${ }^{\mathrm{a}}$

Child Characteristics

Age (months)

Gender

.18

Race

$-.13$

Respondent Characteristics

Age (years)

Child Medical and Immunization History

Child distress at first injection $\quad-.04$

Parent distress at child's first injection $\quad-.13$

Child overall past medical distress $\quad-.11$

Number of medical visits in past 6 months $\quad .24$

Time since last medical visit $\quad-.16$

Number of injections received today $\quad .01$

Travel time to medical clinic $\quad .14$

Family Characteristics

Number of persons living at home $\quad-.01$

Parents' marital status $\quad .07$

(table continues) 


\begin{tabular}{ll}
\hline \multicolumn{1}{c}{ Demographic Variable } & Attendance at Follow-Up Visit \\
\hline Total Annual Family Income & $.32^{*}$ \\
Education of Father & -.02 \\
Education of Mother & -.13 \\
Occupation of father & -.09 \\
Occupation of mother & -.01
\end{tabular}

\footnotetext{
${ }^{a}$ Higher scores indicate greater attendance at follow-up visit

$* p<.05$.
} 
Table 26

Hierarchical Logistic Regression Analysis Predicting Attendance at Follow-Up $\underline{\text { Immunization Visit by MBPS Injection Score }}$

\begin{tabular}{|c|c|c|c|c|c|}
\hline Predictor Variable & $\mathrm{B}$ & SE & Wald & $\mathrm{p}$ & $\begin{array}{l}\text { Odds } \\
\text { Ratio }\end{array}$ \\
\hline \multicolumn{6}{|l|}{ Block 1} \\
\hline Number of people in home & -.27 & .34 & .62 & .43 & .76 \\
\hline Total annual family income & -1.20 & .66 & 3.23 & .07 & .30 \\
\hline Education of father & -.62 & .48 & 1.67 & .20 & .54 \\
\hline Education of mother & .82 & .60 & 1.87 & .17 & 2.28 \\
\hline Child race & .29 & .28 & 1.02 & .31 & 1.33 \\
\hline $\begin{array}{l}\text { Travel time to medical } \\
\text { clinic }\end{array}$ & .02 & .03 & .58 & .45 & 1.03 \\
\hline \multicolumn{6}{|l|}{ Block 2} \\
\hline MBPS Injection Score & .38 & .83 & .21 & .65 & 1.46 \\
\hline
\end{tabular}


Table 27

Hierarchical Logistic Regression Analysis Predicting Attendance at Follow-Up $\underline{\text { Immunization Visit by Parent Rating of Child Distress }}$

\begin{tabular}{|c|c|c|c|c|c|}
\hline Predictor Variable & $\mathrm{B}$ & SE & Wald & $\mathrm{p}$ & $\begin{array}{l}\text { Odds } \\
\text { Ratio }\end{array}$ \\
\hline \multicolumn{6}{|l|}{ Block 1} \\
\hline Number of people in home & -.16 & .33 & .23 & .63 & .86 \\
\hline Total annual family income & -.81 & .56 & 2.06 & .15 & .45 \\
\hline Education of father & -.69 & .49 & 1.96 & .16 & .50 \\
\hline Education of mother & .72 & .59 & 1.48 & .22 & 2.06 \\
\hline Child race & .24 & .27 & .82 & .37 & 1.28 \\
\hline $\begin{array}{l}\text { Travel time to medical } \\
\text { clinic }\end{array}$ & -.001 & .03 & .000 & .98 & 1.0 \\
\hline \multicolumn{6}{|l|}{ Block 2} \\
\hline $\begin{array}{l}\text { Parent rating of child } \\
\text { distress }\end{array}$ & -.09 & .20 & .19 & .67 & .92 \\
\hline
\end{tabular}


Table 28

Hierarchical Logistic Regression Analysis Predicting Attendance at Follow-Up $\underline{\text { Immunization Visit by Nurse Rating of Child Distress }}$

\begin{tabular}{|c|c|c|c|c|c|}
\hline Predictor Variable & $\mathrm{B}$ & SE & Wald & $\mathrm{p}$ & $\begin{array}{l}\text { Odds } \\
\text { Ratio }\end{array}$ \\
\hline Block 1 & & & & & \\
\hline Number of people in home & -.16 & .33 & .23 & .63 & .86 \\
\hline Total annual family income & -.81 & .56 & 2.06 & .15 & .45 \\
\hline Education of father & -.69 & .49 & 1.96 & .16 & .50 \\
\hline Education of mother & .72 & .59 & 1.48 & .22 & 2.06 \\
\hline Child race & .24 & .27 & .82 & .37 & 1.28 \\
\hline $\begin{array}{l}\text { Travel time to medical } \\
\text { clinic }\end{array}$ & -.001 & .03 & .000 & .98 & 1.0 \\
\hline Block 2 & & & & & \\
\hline $\begin{array}{l}\text { Nurse rating of child } \\
\text { distress }\end{array}$ & -.04 & .14 & .10 & .76 & .96 \\
\hline
\end{tabular}




\section{Figure Captions}

Figure 1. Interaction between prior medical experience and adherence to immunization schedules: MBPS injection score.

Figure 2. Interaction between health care attitudes and adherence to immunization schedules:

MBPS injection score. 


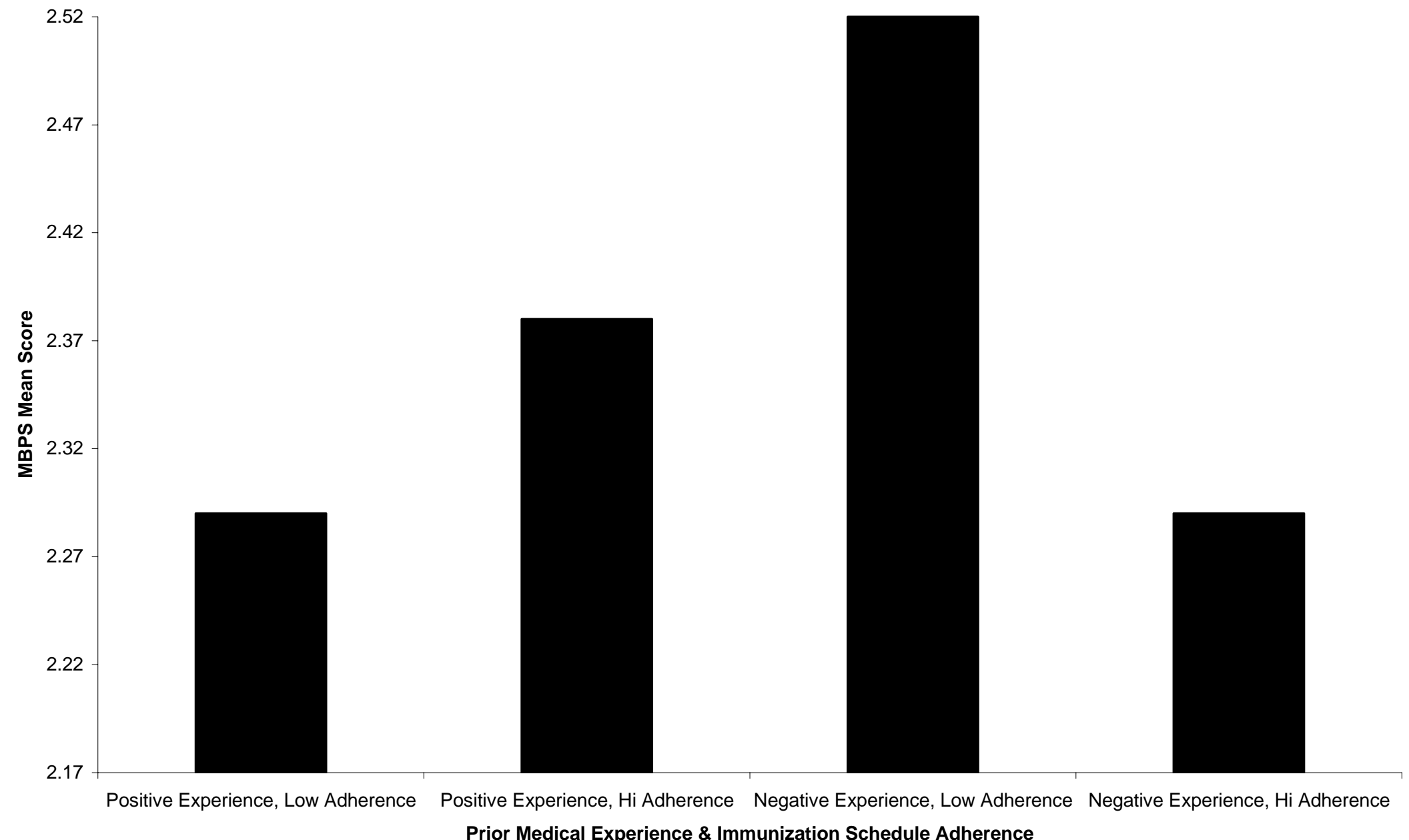




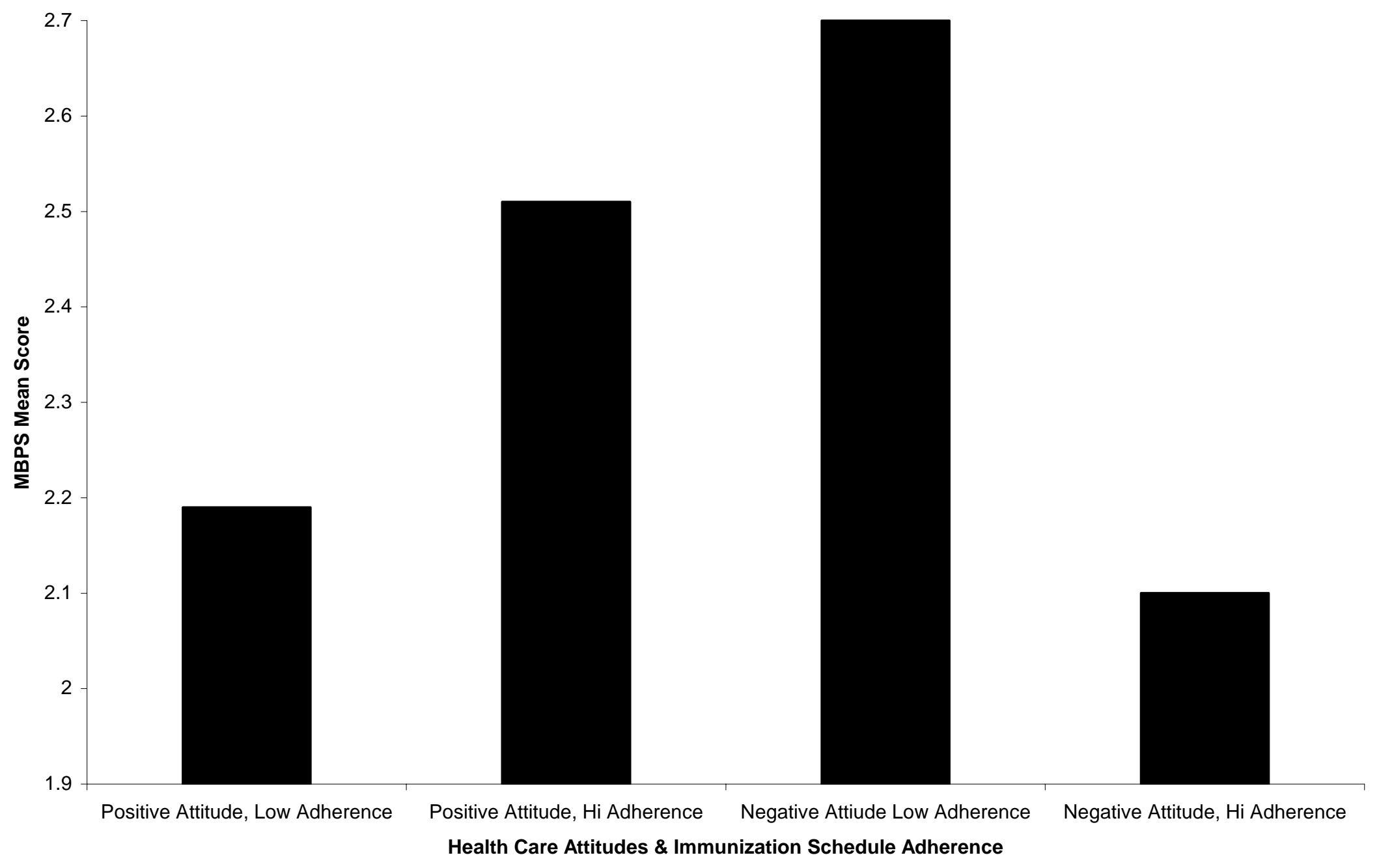

